ARTICLE

\title{
Genetically encoded formaldehyde sensors inspired by a protein intra-helical crosslinking reaction
}

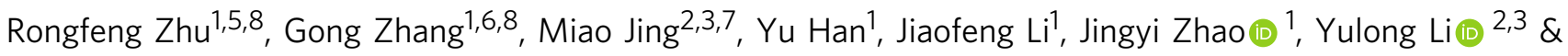
Peng R. Chen (1) $1,2,4 \bowtie$

Formaldehyde (FA) has long been considered as a toxin and carcinogen due to its damaging effects to biological macromolecules, but its beneficial roles have been increasingly appreciated lately. Real-time monitoring of this reactive molecule in living systems is highly desired in order to decipher its physiological and/or pathological functions, but a genetically encoded FA sensor is currently lacking. We herein adopt a structure-based study of the underlying mechanism of the FA-responsive transcription factor HxIR from Bacillus subtilis, which shows that $\mathrm{HxIR}$ recognizes $\mathrm{FA}$ through an intra-helical cysteine-lysine crosslinking reaction at its $\mathrm{N}$-terminal helix $\alpha 1$, leading to conformational change and transcriptional activation. By leveraging this FA-induced intra-helical crosslinking and gain-of-function reorganization, we develop the genetically encoded, reaction-based FA sensor-FAsor, allowing spatial-temporal visualization of $\mathrm{FA}$ in mammalian cells and mouse brain tissues.

\footnotetext{
${ }^{1}$ Synthetic and Functional Biomolecules Center, Beijing National Laboratory for Molecular Sciences, College of Chemistry and Molecular Engineering, Peking University, 100871 Beijing, China. ${ }^{2}$ Peking-Tsinghua Center for Life Sciences, 100871 Beijing, China. ${ }^{3}$ State Key Laboratory of Membrane Biology, PKU-IDG/McGovern Institute for Brain Research, School of Life Sciences, Peking University, 100871 Beijing, China. ${ }^{4}$ Key Laboratory of Bioorganic Chemistry and Molecular Engineering of Ministry of Education, Peking University, 100871 Beijing, China. ${ }^{5}$ Present address: State Key Laboratory of Cellular Stress Biology, Innovation Center for Cell Signaling Network, School of Life Sciences, Xiamen University, 361005 Xiamen, Fujian, China. ${ }^{6}$ Present address: Chongqing Key Laboratory of Natural Product Synthesis and Drug Research, School of Pharmaceutical Sciences, Chongqing University, 401331 Chongqing, China. ${ }^{7}$ Present address: Chinese Institute for Brain Research, 102206 Beijing, China. ${ }^{8}$ These authors contributed equally: Rongfeng Zhu, Gong Zhang. ${ }^{{ }_{e}}$ email: pengchen@pku.edu.cn
} 
$\mathrm{n}$ addition to its broad presence in environment, various cellular processes such as the one-carbon metabolism and the oxidative demethylation on proteins and nucleic acids produce formaldehyde $(\mathrm{FA})^{1-3}$. As the simplest reactive carbonyl species (RCS), the cytotoxicity of FA has been well documented due to its high reactivity with various biological nucleophiles ${ }^{4-6}$. Meanwhile, the high physiological abundance of FA (e.g., $\sim 0.1 \mathrm{mM}$ in blood, and 2-4 times higher in liver and nasal mucosa) indicates potential physiological effects that have been increasingly appreciated $^{7-9}$. For example, low concentration of FA is reported to increase human melanoma cell proliferation and MAPK pathway activation $^{10,11}$. Additionally, FA is found to support the survival of one-carbon-cycle-defective cells recently ${ }^{12}$. In methylotrophic bacteria, FA even serve as a sole carbon source, being assimilated via ribulose monophosphate (RuMP) pathway ${ }^{13}$. A major challenge in deciphering the biological roles of FA is the real-time measurement of its concentration, duration, and location in living cells and tissues. Although FA is an abundant molecule in vivo, stringent protection mechanisms have been evolved to scavenge this highly reactive RCS with little knowledge about its intracellular distribution ${ }^{14-16}$. Fluorescent sensors represent a powerful noninvasive tool for real-time monitoring of endogenous FA organization in space and time and small molecule-based FA sensors have been elegantly developed in recent years ${ }^{17-22}$. Alternatively, genetically encoded, fluorescent protein (FP)-based sensors are advantageous for subcellular targeting and cell-typespecific expression as well as potential long-term imaging in animals ${ }^{23-25}$. However, no such genetically encoded FA sensors are currently available.

A major hurdle is due to the lack of knowledge and molecular details on whether and how a protein can actively recognize and transduce the "signal" from a highly reactive FA molecule, which could be converted into a fluorescence change on the coupled $\mathrm{FPs}^{26}$. To this end, we first study the structural and molecular mechanism of a FA-responsive transcription factor $\mathrm{HxlR}^{27}$, which reveals a FA-triggered crosslinking reaction between the sidechains of residues Cys 11 and Lys13 on helix $\alpha 1$. The resulting intrahelical methylene bridge (Cys- $\mathrm{CH}_{2}$-Lys) represents a protein modification with a conformational change that allosterically induced HxlR's transcriptional activation. Inspired by the unique gain-of-function FA-sensing mechanism of HxlR, we convert its intrahelical crosslinking-induced conformational change into a fluorescence change of the inserted circularly permutated yellow FP (cpYFP). This genetically encoded FA sensor-FAsor allows direct visualization of FA in diverse living species including mammalian cells as well as the brain tissue of mice.

\section{Results}

FA directly activates HxlR via a gain-of-function, intrahelical crosslinking reaction. Among the currently reported putative FA-responsive proteins we surveyed, Bacillus subtilis HxlR is particularly intriguing because this MarR/DUF24 family transcriptional regulator "positively" responds to FA with an enhanced transcription activity ${ }^{27,28}$ (Supplementary Note 1, Supplementary Fig. 1a). Such a gain-of-function response implies an active recognition and transducing mechanism of the FA signal by HxlR. In contrast, for the FA transcriptional factors that are inactivated by FA, it is difficult to exclude the possibility of damaging effects as opposed to specific recognition ${ }^{26,29,30}$. We thus decided to adopt a structure-based approach to study this positive FA regulator.

We first solved the crystal structures of HxlR with and without FA. After optimizing the DNA sequence, we successfully crystallized the HxlR-DNA complex after FA treatment and the resulting structure, HxlR-WT-FA-DNA, was solved to 2.9- resolution (Fig. 1a and Supplementary Table 1, Supplementary Note 2). The crystal structure of wild-type HxlR without DNA and FA treatment (HxlR-WT) was also solved to 2.6- $\AA$ resolution (Fig. $1 \mathrm{~b}$ and Supplementary Table 1). Both FA-treated and -untreated HxlR adopt an overall topology similar to the previously reported MarR/DUF24 family proteins that bind DNA in a dimeric form, with each monomer consisting of dimerization domain (helices $\alpha 1$ and $\alpha 5$ ) and winged helix-turnhelix (wHTH) DNA-binding domain (helices $\alpha 2, \alpha 3$, $\alpha 4$, and $\beta$ strands $)^{31-33}$. The distance between the DNA-binding helices $\alpha 4$ and $\alpha 4^{\prime}$ were $34.8 \AA$ in HxlR-WT-FA-DNA and 44.0 $\AA$ in HxlRWT, consistent with the DNA-bound and non-DNA-bound forms of MarR/DUF24 family proteins, respectively (Fig. 1a). Interestingly, a covalent bridge was clearly visible between the side-chains of Cys11 and Lys13 on the $2 F_{\mathrm{o}}-F_{\mathrm{c}}$ electron density map of the HxlR-WT-FA-DNA structure (Fig. 1a), while the sidechains of Cys11 and Lys13 were located oppositely in helix $\alpha 1$ and their side-chains were separated $9.6 \AA$ away from each other in the HxlR-WT structure (Fig. 1b). Further refinement of the HxlR-WT-FA-DNA structure modeled a methylene bridge connecting the thiol group on Cys11 and the amine group on Lys13 (Cys- $\mathrm{CH}_{2}$-Lys; Fig. 1a). Particularly, Cys11 is a conserved residue and has been reported to react and sense electrophiles and/or oxidants in other MarR/DUF24 family members (Supplementary Fig. 1b) ${ }^{32-34}$. We thus speculated that HxlR may react with FA via Cys11 and Lys13, and the resulting intrahelical methylene bridge and conformational changes may allosterically regulate its DNA-binding domain.

In order to verify our speculations, we first used mass spectrometry (MS) to validate the FA-induced methylene bridge between $\mathrm{N}$-terminal Cys11 and Lys13 observed in the crystal structure. Our FT-MS analysis of the purified HxlR protein before and after FA treatment showed a newly formed $+12 \mathrm{Da}$ mass peak in the FA-treated sample, corresponding to formation of a methylene bridge in the protein (Supplementary Fig. 1c). Next, we stimulated the HxlR-expressing cells with FA and performed MS-MS analysis on the purified HxlR protein. Consistent with the results on the purified protein, a $+12 \mathrm{Da}$ mass deviation was found on MS1 scan of the peptide containing Cys11 and Lys13, and the MS2 spectra of the same peptide further verified a methylene bridge formation between these two residues (Fig. 1c). Finally, we verified the FA-induced Cys11-Lys13 crosslinking reaction and the methylene bridge formation via a synthetic peptide containing the native residues from Phe9 to Leu15 on HxlR protein (Ac-FNCEKEL- $\mathrm{NH}_{2}$ ). The newly formed $+12 \mathrm{Da}$ mass peak on the peptide after FA incubation, which was absent in the peptides with Ala replacing Cys11 or Lys13, confirmed that FA is able to induce intramolecular crosslinking with a methylene bridge formation between Cys11 and Lys13 in this peptide (Fig. 1d, Supplementary Note 3 and Supplementary Fig. 1d).

We next investigated whether the FA-triggered intrahelical crosslinking reaction could lead to HxlR activation. First, we performed an in vitro electrophoretic mobility shift assay (EMSA) to compare HxlR's DNA-binding ability with and without FA treatment. The results showed that incubating HxlR-WT with 0.6 mM FA was able to enhance its DNA affinity (Fig. 1e). We then generated HxlR mutants with Cys11 mutated to Ala (HxlRC11A), and utilized the same assay to evaluate the effect of FA on their DNA binding. Different from HxlR-WT, HxlR-C11A mutant exhibited little change after incubation with the same amount of FA (Fig. 1e), indicating that FA can specifically activate HxlR through the intrahelical Cys-Lys crosslinking reaction. We next validated this FA-induced HxlR activation in vivo by monitoring the transcription activity. A HxlR-inducible GFP reporter encoding the $g f p$ gene under the control of $h x l A B$ promoter $(h x l A B-g f p)$ was transformed into the $E$. coli 
a
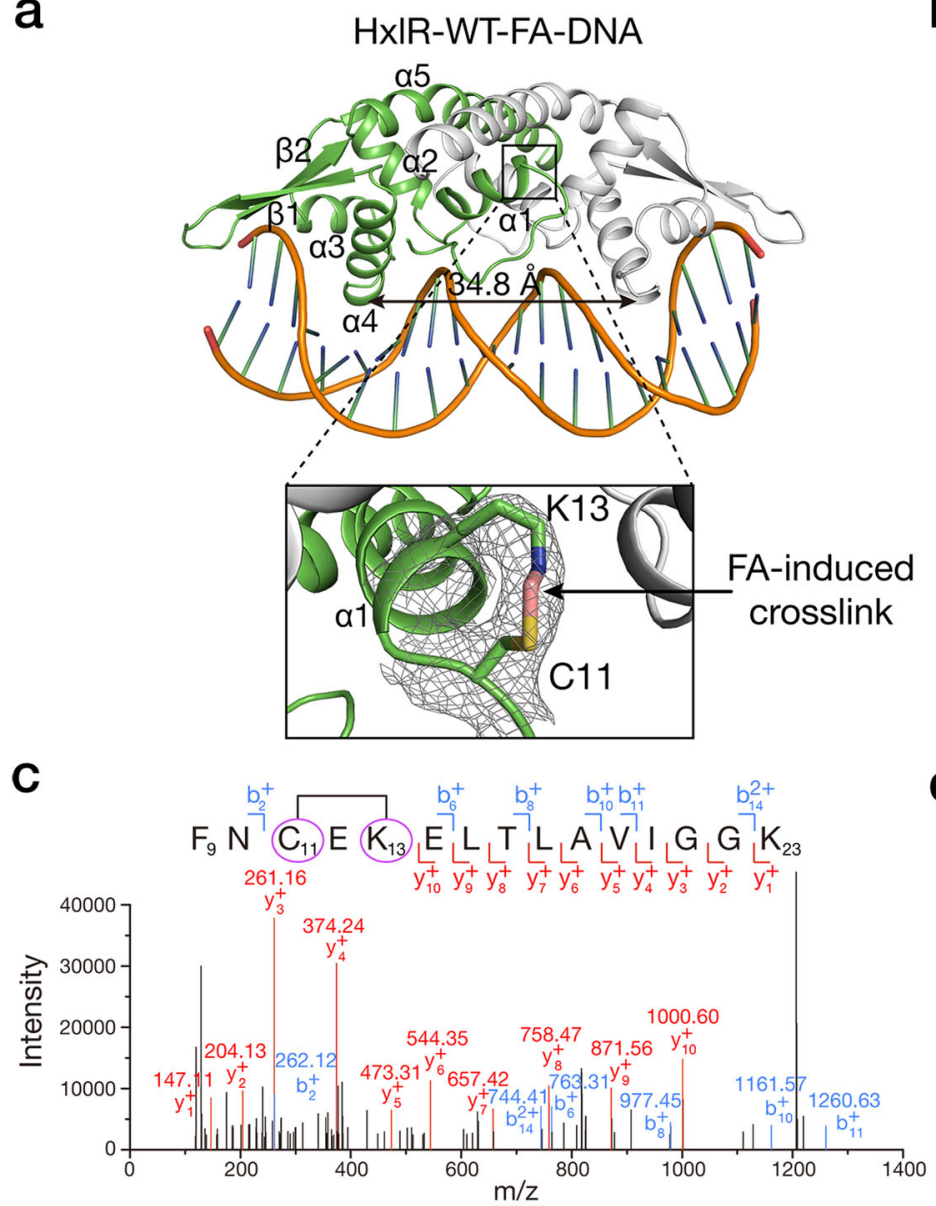

e

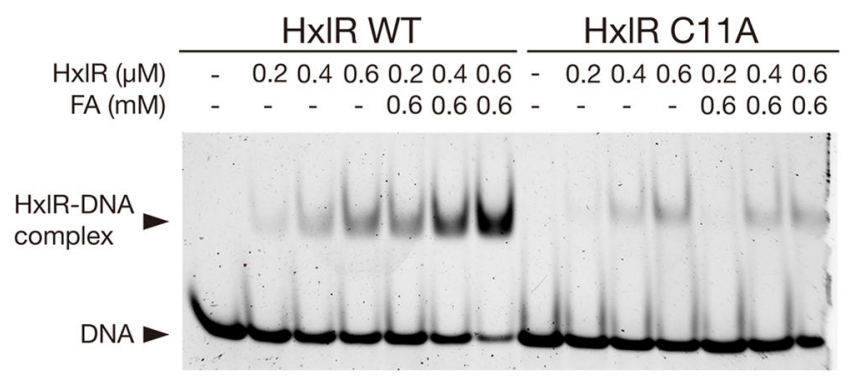

b

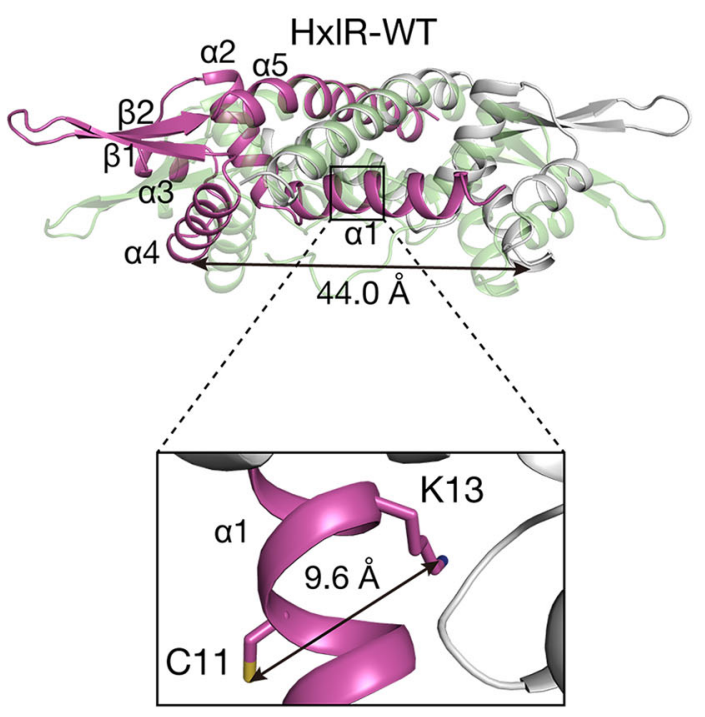

d

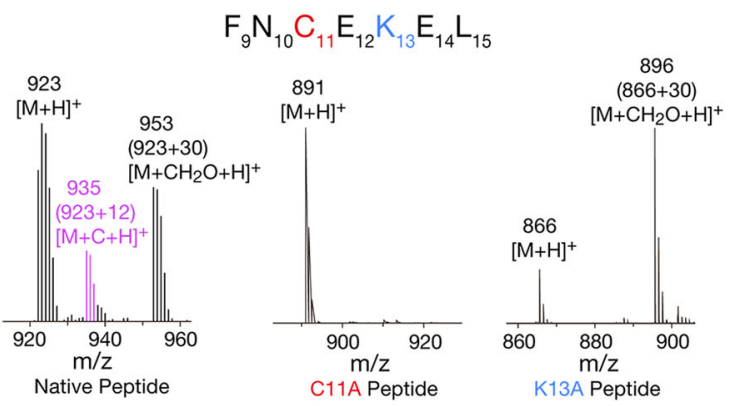

f

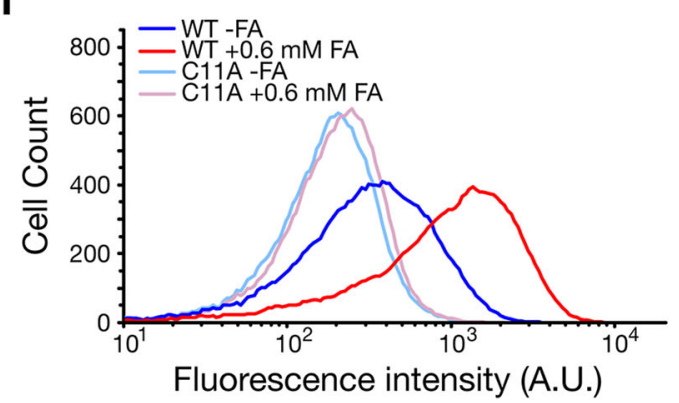

Fig. 1 HxIR directly senses FA via the intrahelical Cys-Lys crosslinking reaction. a Crystal structure of FA-activated HxIR protein/DNA complex (HxIRWT-FA-DNA). Close-up view (right) shows the FA-triggered crosslinking reaction between residues Cys 11 and Lys 13 on helix $\alpha 1$. The $2 F_{\mathrm{o}}-F_{\mathrm{c}}$ electron density map contoured at $1 \sigma$ indicates that an intrahelical methylene bridge (pink) was formed between Cys 11 and Lys 13 upon FA treatment. b Crystal structure of HxIR protein in the absence of FA (HxIR-WT). Close-up view (right) shows the side-chains of residues Cys 11 and Lys 13 which face opposite directions. The distance between the thiol group on Cys11 and the amine group on Lys13 is $9.6 \AA$, indicating no interactions between these two residues. Superposed structures of HxIR-WT and HxIR-WT-FA-DNA (light green) showed obvious conformational change with FA-induced intrahelical crosslinking reaction. c MS-MS characterization of FA-induced methylene bridge between Cys11 and Lys13 on intact HxIR protein under living conditions. In the MS/MS spectrum of a peptide F9-K23, the fragment ions b2 ${ }^{+}$and y10 ${ }^{+}$correspond to the peptide fragments ${ }_{9} \mathrm{FN}_{10}$ and ${ }_{14}$ ELTLAVIGGK $_{23}$, respectively (observed $\mathrm{m} / z 262.12$ and 1000.60), which verified the methylene bridge formation between Cys11 and Lys13 (+12 Da, highlighted by purple circles and the black line between them). $\mathbf{d}$ LC-MS analysis verifying the FA-induced methylene bridge between Cys11 and Lys13 on a peptide sequence derived from HxIR's helix $\alpha 1$. Mutation of either Cys11 or Lys13 abolished the methylene bridge formation (+12 Da, the $[\mathrm{M}+\mathrm{C}+\mathrm{H}]^{+}$ion). e Cys11 is essential for FA-enhanced DNA binding of HxIR. Determination of HxIR's DNA-binding ability via EMSA showed that 0.6 mM FA was able to increase DNA-binding ability of HxIR-WT protein, whereas little change was observed for the HxIR-C11A mutant, in the presence of FA. f Flow cytometric analysis of the $h x / A B-g f p$ reporter-harbored E. coli BW25113 cells expressing HxIR-WT or HxIR-C11A proteins with and without FA treatment. The addition of $0.6 \mathrm{mM} F A$ increased the expression level of GFP in the bacterial strain expressing HxIR-WT, while little change was observed on the bacterial strain expressing HxIR-C11A, indicating that Cys11 is essential for FA-enhanced transcription activity of HxIR in vivo. 
BW25113 strains expressing HxlR-WT and HxlR-C11A, respectively (Supplementary Fig. 2a) ${ }^{27}$. Having confirmed that FA affected viability of bacteria little in the measurement, we treated the bacterial cells with or without FA, followed with analyzing the viability and HxlR-regulated transcription activity via flow cytometry (Supplementary Fig. 2b, c). Indeed, FA was able to increase $g f p$ expression under the control of HxlR-WT, but not HxlR-C11A mutant (Fig. 1f). In addition, we measured the effect of K13A mutation on HxlR's DNA binding and transcriptional regulation. Although with a higher basal level on DNA binding and transcriptional activation, the K13A mutant (HxlR-K13A) also remained insensitive to FA (Supplementary Fig. 2d, e). Taken together, we confirmed that HxlR uses a gain-of-function, intrahelical crosslinking mechanism between its $\mathrm{N}$-terminal residues Cys11 and Lys13 to sense FA and trigger the transcriptional responses.

Structural bases for FA-induced HxlR activation. In order to further understand how HxlR protein is activated via the FAtriggered intrahelical crosslinking reaction, we next closely inspected the difference between the structures of HxlR-WT and HxlR-WT-FA-DNA (Supplementary Note 4, Supplementary Fig. 3a-e). When the dimeric form of HxlR-WT-FA-DNA and HxlR-WT were superimposed on one monomer, the nonsuperimposed subunits showed a large conformational change, with the wHTH domain rotated 21.9 degree and tips of the $\beta$ wings translocated $24.6 \AA$ (Fig. 2a). It is noteworthy that although the superimposed subunits of HxlR-WT-FA-DNA and HxlR-WT fit close to each other (r.m.s.d. $=0.595 \AA$ for $82 \mathrm{Ca}$ atoms, Supplementary Fig. 3f), an obvious difference was observed on the $\mathrm{N}$ terminal residues. Whereas the $\mathrm{N}$-terminal residues formed an intact helix al in the HxlR-WT structure, this helix breaks at Cys11 in HxlR-WT-FA-DNA, with residues before Cys11 becoming unstructured. This helix breakage is likely due to the FA-triggered Cys11-Lys13 crosslinking reaction, which produced a covalent methylene bridge between these two residues that were originally separated by $9.6 \AA$ and located on the opposite sides of helix a1. In order to accommodate this newly formed intrahelical methylene bridge, helix a1 was forced to break at Cys 11 and rotate $\sim 120$-degree that caused the $\mathrm{N}$-terminal residues to flip away from the original orientation (we termed this as "N-terminal helix-flipping", Fig. 2a, b).

We next investigated the relationships between this local " $\mathrm{N}$ terminal helix-flipping" and the global conformational change on HxlR. Several MarR/DUF24 family members have been reported to undergo signal-induced local conformational change at their dimer interface that allosterically regulates DNA binding 32,33 . Similarly, close inspections on HxlR's dimer interface indicated that the "N-terminal helix-flipping" slightly twisted helix a5" towards helix a1, with the sidechain of Tyr99' now filled in the space originally occupied by helix al before breakage (Fig. 2c). The other helix in the dimerization domain-helix a2' now rotate accordingly to maintain its interactions with helix a5' (e.g., the electrostatic interactions between Lys34' and Glu96', Fig. 2c), which further rotate the wHTH domain. Meanwhile, it should be noted that rotation of Lys13 upon crosslinking with Cys11 also promoted the rotation of helix a2', with the side-chain of Met26' now filled in the space originally occupied by Lys13 (Fig. 2d). Consequently, FA-triggered intrahelical crosslinking and " $\mathrm{N}$ terminal helix-flipping" induced a coordinated rotation and movement of helices at HxlR's dimer interface, which allosterically rearranged its wHTH domains to adopt a more optimal conformation for DNA binding.

Further details of the "N-terminal helix-flipping" mechanism was revealed with structure of HxlR-K13A. The 1.7 - $\AA$-resolution
HxlR-K13A structure exhibited a more optimal conformation for DNA binding than HxlR-WT but had less significant changes than that of HxlR-WT-FA-DNA (Supplementary Note 5, Supplementary Fig. $3 g$ and Supplementary Table 1). Particularly, helix a 1 does not break at Cys11 in the HxlR-K13A structure and residues Cys11 and Ala13 are located on the opposite sides of an intact helix $\alpha 1$ (Fig. 2e), indicating that the absence of "N-terminal helix-flipping" might be the main reason why HxlRK13A does not undertake the conformation as HxlR-WT-FADNA. Further inspection at the N-terminus of HxlR-K13A revealed that the sidechain of Phe9 is located between Trp29' and 'Tyr99' on the dimer interface (Fig. 2f). In contrast, Phe9 is on the opposite side of helix al due to the $\mathrm{N}$-terminal helix-flipping on the HxlR-WT-FA-DNA structure, which shortened the distance between the rings of Trp29' and Tyr99' from $5.3 \AA$ to $4.0 \AA$ (Fig. 2f). Therefore, the translocation of Phe9 together with the movement of helices $\alpha 2$ ' and a5' supported the key role of the "N-terminal helix-flipping" in enforcing HxlR's helices a1, a2', and $\alpha 5$ ' closer to each other, resulting in an optimal conformation for DNA binding. In the absence of the "N-terminal helixflipping", HxlR-K13A can only adopt an intermediate conformation between that of HxlR-WT and HxlR-WT-FA-DNA.

Therefore, we propose the structural mechanism of FA-triggered HxlR activation as follows: (1) FA crosslinks the sidechain of Cys11 and Lys13 in HxlR protein to produce an intrahelical methylene bridge; (2) This methylene crosslink triggers the "N-terminal helixflipping" that forces the mainchain of residues in front of Cys11 to extend away from helices $\alpha 2$ ' and $\alpha 5$ '; (3) The sidechain of Lys13 also moves away from helix $\alpha 2^{\prime}$, allowing the movement of helices $\alpha 1, \alpha 2^{\prime}$, and $\alpha 5^{\prime}$ at the dimer interface; (4) Rotation and translocation of helices $\alpha 2$ ' and $\alpha 5^{\prime}$ further drives the movement of the whole wHTH domain, decreases the distance of helices a4 and $a 4^{\prime}$, and induces HxlR to undertake an optimal conformation for DNA binding.

Mechanism-inspired design and characterization of the genetically encoded FA sensors. Inspired by this unique FA-sensing mechanism, we set out to develop a genetically encoded formaldehyde sensor by coupling the FA-induced conformational change of HxlR with local-environment-dependent fluorescence response of circularly permuted fluorescence proteins (cpFPs) ${ }^{35-37}$. Since the direction of Phe9 on helix al turned 50 degree upon FA treatment, we chose to link two HxlR monomers through cpYFP between helices $\alpha 5$ and $\alpha 1$ ' (Fig. 3a). A total of nine constructions were created and all of the resulting HxlR-cpYFP-HxlR fusion proteins (HYH) exhibited dosedependent fluorescence responses to FA despite of different inserted sites (Supplementary Fig. 4a-1). In contrast, control experiments using cpYFP alone showed little change of fluorescence, suggesting that the fluorescence change was not caused by the perturbation of the chromophore (Supplementary Fig. 4c). Furthermore, the FA-induced conformational change happened in the absence of HxlR's cognate DNA, which means that the optimal DNA-binding conformation of HxlR in the HxlR-WTFA-DNA was not caused by the induced-fit effect of its cognate DNA. These results indicated that insertion of cpYFP between HxlR monomers is able to form a genetically encoded FA sensor. Encouraged by this initial attempt, we then optimized the performance by systematically varying the linker sequence between cpYFP and HxlR. Among all the variants we tested, the HYH-5 variant exhibited a ratiometric property, with an increased fluorescence response to FA when excited above $467 \mathrm{~nm}$, and decreased fluorescence response below $467 \mathrm{~nm}$, while all other constructions showed intensiometric decreased fluorescence response (Supplementary Fig. 4d-1). Therefore, the HYH-5 
a

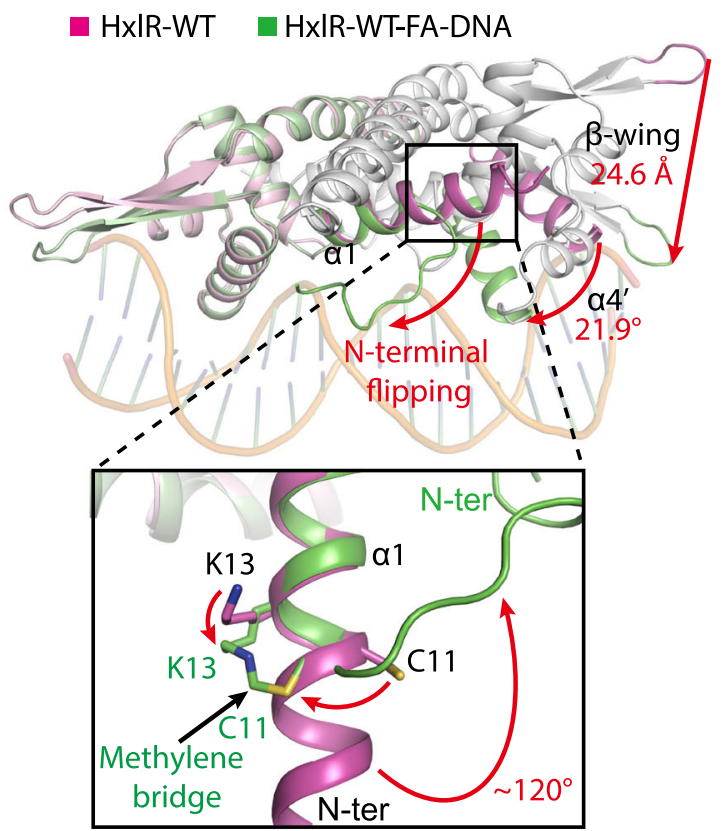

C

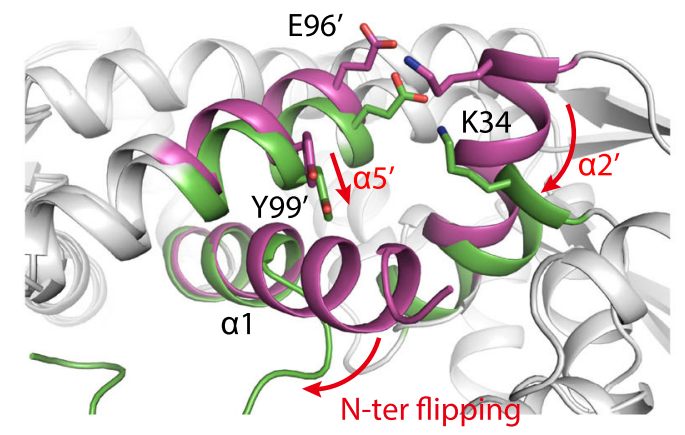

e

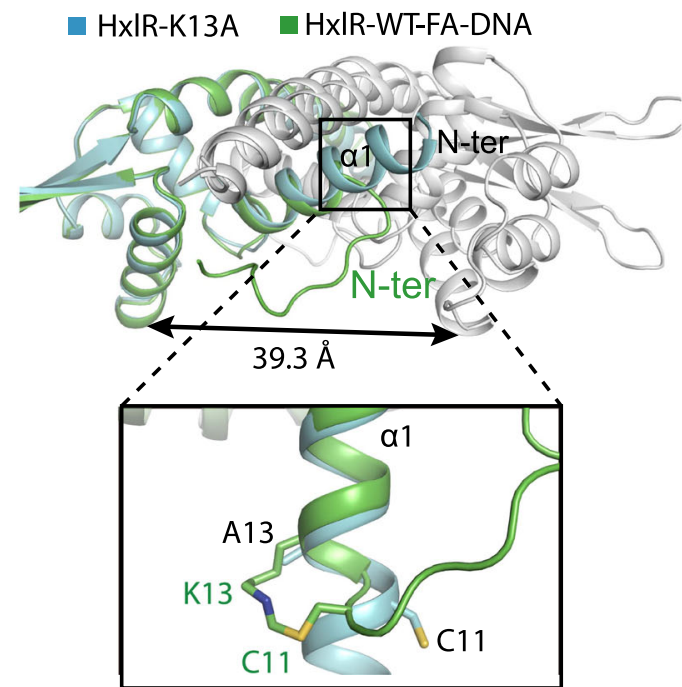

variant, which has cpYFP inserted between residues Tyr105 and Phe9' of the two HxlR monomers and a Glu-Phe sequence spacer before the N-terminus of cpYFP, was selected and renamed as FAsor (FA sensor) for further investigations.

The ratiometric property of FAsor is highly desired for quantitative detection of analytes in living cells. We first titrated b

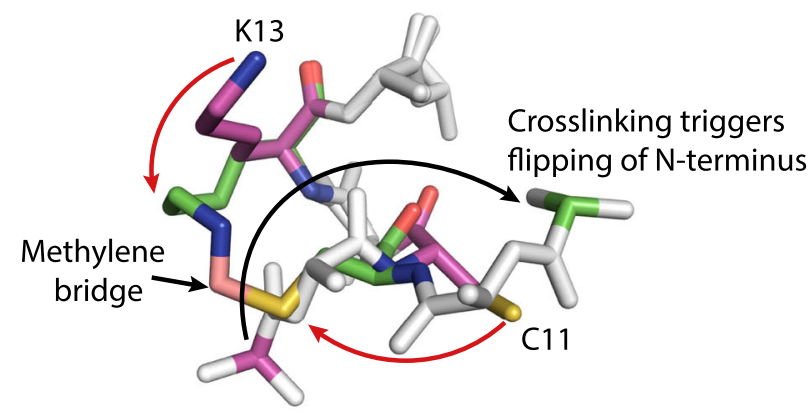

Rotation of C-C a bond in Cys11

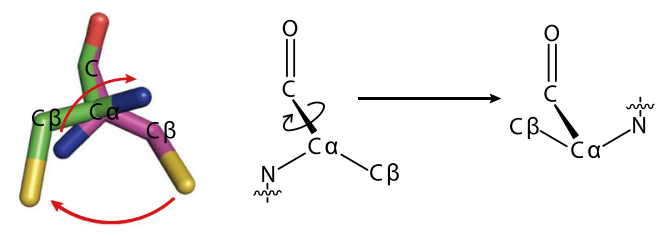

d

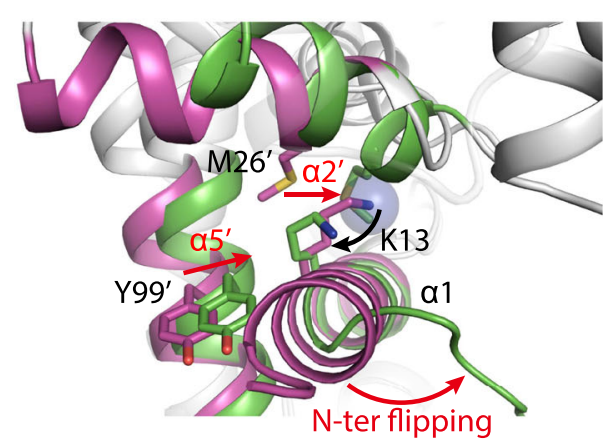

$f$

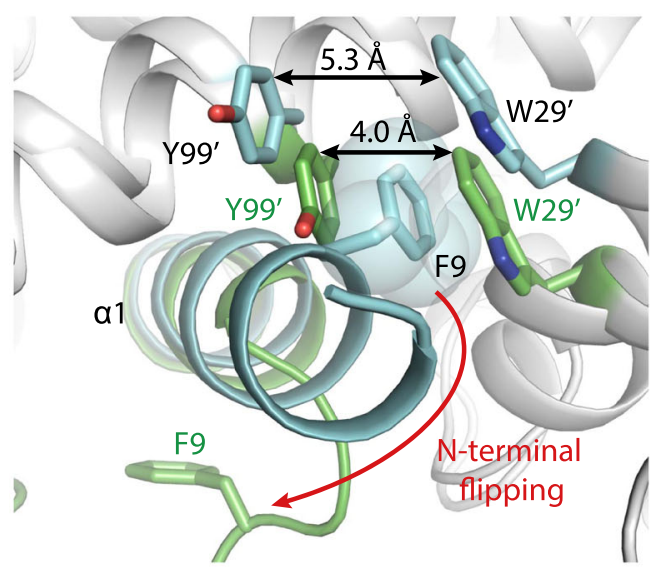

FAsor with serial concentrations of FA ranging from 0 to $5 \mathrm{mM}$. Results revealed that FAsor exhibited a dose-dependent fluorescence response to FA, with an approximately 2.2 -fold enhanced signal at $2 \mathrm{mM}$, and a detectable response to $\mathrm{FA}$ at a concentration as low as $20 \mu \mathrm{M}$ (Fig. 3b,c). As for negative control, we introduced the Cys11-to-Ala or Lys13-to-Ala mutation to both HxlR 
Fig. 2 Structural bases for FA-induced HxIR activation. a The "N-terminal helix-flipping" mechanism of HxIR protein upon FA treatment. Superposition of HxIR-WT (magenta) and HxIR-WT-FA-DNA (green) indicates that FA induces a dramatic rearrangement on helix $\alpha 1$. The FA-triggered Cys11-Lys13 intrahelical crosslinking and methylene bridge formation forced the residues before Cys 11 to flip $\sim 120$ degree away from the original orientation, and become unstructured. This " $\mathrm{N}$-terminal helix-flipping" caused a large movement of the wHTH domain, with helix $\alpha 4$ rotated 21.9 degree and the tips of $\beta$ wings translocated $24.6 \AA$, producing an optimized conformation for DNA binding. Translocations of the structure domains and residues are indicated by the red arrow. b Detailed view of the crosslinking-induced "N-terminal helix-flipping". In order to form the crosslink, C-C $\alpha$ bond in Cys 11 rotated to allow the sidechain thiol group to get close to Lys13 (red arrow), leading the $\mathrm{N}$ atom of Cys11 and the connected $\mathrm{N}$-terminal residues to flip to the opposite side (black arrow). c The "N-terminal helix-flipping" triggered the reorganization of helices $\alpha 1, \alpha 2$ ', and $\alpha 5$ ', with the residues before Cys 11 becoming unstructured, helix $\alpha 5^{\prime}$ moving towards helix $\alpha 1$ and the concurrent rotation of helix $\alpha 2^{\prime}$. Translocations of these helices are indicated by red arrows. $\mathbf{d}$ The FA-induced Cys11-Lys13 intrahelical crosslinking brings helix $\alpha 2^{\prime}$ towards helices $\alpha 1$. The resulting methylene bridge forced helix $\alpha 2^{\prime}$ to move closer to helix $\alpha 1$, with Met26' filling the space left by Cys11-Lys13 crosslinking (shown in sphere). Red arrows indicate the movement of Lys13 and helix $\alpha 2^{\prime}$.

e Superposition of HxIR-K13A (cyan) and HxIR-WT-FA-DNA (green) indicates the absence of "N-terminal helix-flipping" in HxIR-K13A. Similar to that of HxIR-WT, helix $\alpha 1$ in HxIR-K13A extends beyond Cys11 to the N-terminal residues, with Cys11 and Ala13 located on the opposite sides. Distances between the DNA-binding helices $\alpha 4$ and $\alpha 4^{\prime}$ are indicated by black arrow. Residues from HxIR-K13A and HxIR-WT are marked in black and green, respectively. f "N-terminal helix-flipping" is crucial for HxIR to undertake the DNA-binding conformation. As the "N-terminal helix-flipping" does not occur in the HxIRK13A mutant, the sidechain of Phe9 is located between that of Trp29' and Tyr99', which causes the distance between Trp29' and Tyr99' larger than that of HxIR-WT-FA-DNA and prevents further approaching of HxIR subunits. The sidechain of Phe9 is shown in sphere, and the distances between Trp29' and Tyr99' in different conformations are indicated by black arrows.

monomer proteins in FAsor. The resulting variants FAsor-C11A and FAsor-K13A had no response to FA up to $5 \mathrm{mM}$ (Fig. $3 \mathrm{c}$ ). These results together proved that both Cys11 and Lys13 are essential for FA-induced conformational change on HxlR protein, and ruled out the perturbance of the chromophore by environment changes or other damaging effects. Following kinetic characterization showed that the FAsor had a rapid response to FA treatment with $>80 \%$ change occurred within $5 \mathrm{~min}$ (Fig. $3 \mathrm{~d}$ ), which is consistent with the time-scale for HxlR-regulated transcriptional activation in vivo ${ }^{38}$. Consistent with the essential roles of Cys11 and Lys13 for FA sensing, both FAsor-C11A and FAsor-K13A showed negligible change after addition of $0.5 \mathrm{mM}$ FA during the entire measurement (Fig. 3d). Considering the sensitivity of cpYFP to $\mathrm{pH}^{35}$, we next investigated the effects of buffer $\mathrm{pH}$ on fluorescence properties. FA is able to induce fluorescence change of FAsor-WT in buffers with $\mathrm{pH}$ between 6.6 and 8.2, showing that the ratiometric change of FAsor can reliably detect FA within the $\mathrm{pH}$ range (Fig. $3 \mathrm{e}$ ). In contrast, the $F_{503 \mathrm{~nm}} / F_{427 \mathrm{~nm}}$ ratio of FAsor$\mathrm{C} 11 \mathrm{~A}$ did not respond to $\mathrm{FA}$ in the same $\mathrm{pH}$ range (6.6-8.2; Fig. 3e), indicating this variant can thus be utilized as a control probe to rule out the effect of $\mathrm{pH}$ change. The specificity of FAsor was also verified with various carbonyl-containing small molecule metabolites including acetaldehyde (ACA), methylglyoxal (MG), glucose, glucosone, pyruvate, pyridoxal as well as hydrogen peroxide. Most of the tested compounds caused negligible changes of FAsor's fluorescence except for MG, which induced similar response with FA (Fig. 3f). The result was not unprecedented as HxlR has been shown to respond to methylglyoxal in B. subtilis ${ }^{38}$. Yet we consider it has limited effect on in vivo application of FAsor in most cases, as MG has a much lower endogenous concentration than $\mathrm{FA}^{39}$. Notably, despite exhibiting a $20 \%$ decrease of the fluorescence response, FAsor can still sense FA in the presence of 5 $\mathrm{mM}$ glutathione (GSH) (Fig. 3g). Our results indicated that FAsor can preferentially form the covalent crosslinking with FA under this condition, allowing detection of FA in the presence of physiological concentrations of glutathione.

Subcellular visualization of FA by FAsors in living cells. Having established that FAsors can respond to FA in vitro, we next evaluated their ability to sense FA in living cells. Flow cytometry showed an increase of $F_{488 \mathrm{~nm}} / F_{405 \mathrm{~nm}}$ ratio of cells transfected with FAsor-WT upon treatment of exogenous FA (Fig. 4a, Supplementary Fig. 5a). In contrast, cells transfected with FAsor-C11A showed negligible response to FA, indicating that the fluorescence change was induced by FA-induced conformational change of
HxlR but not other changes in cellular environment (Fig. 4a). We next utilized FAsor to visualize subcellular change of FA by confocal microscopy. Different subcellular-targeting sequence, including nucleus, cytoplasm and mitochondria, were fused to FAsor to generate subcellular-targeting FAsor variants, which all showed the desired subcellular localizations (Supplementary Fig. 5b). Imaging with nuclear-, cytosolic- and mitochondrialFAsor in HeLa cells all showed the increased $F_{488 \mathrm{~nm}} / F_{405 \mathrm{~nm}}$ ratiometric change upon FA treatment (Fig. 4b, Supplementary Fig. 5c). As controls, the subcellular-targeting variants of the FAsor-C11A showed no obvious change upon FA (Supplementary Fig. 5d, e). Furthermore, we also simultaneously visualized FA levels in nucleus and cytosol with FAsor variants that have different colors of cpFPs inserted (Supplementary Note 6, Supplementary Fig. 5f, g). Together, these results indicated that the genetically encoded, subcellular-targeted FAsors offered a convenient toolkit for dynamic monitoring of FA levels in different subcellular compartments within living cells.

Visualization of endogenous FA dynamics in living cells by FAsors. Then we turned our attention to investigate the dynamic production of endogenous FA inside cells. FA is continuously produced and consumed and the homeostasis of FA is dynamically regulated inside cells. Since our in vitro measurements indicated that GSH is able to compete with FAsor on reaction with FA, we first investigated the effect of GSH on FAsor signal in cells. We first incubated cells with and without FA, followed by changing to medium with addition of mono-ethyl-GSH, which is membrane-soluble and able to release GSH inside cells. After addition of mono-ethyl-GSH, the FAsor signal dropped to lower than the basal level (Supplementary Fig. 5h, i). These results indicated that GSH is able to reverse the reaction of FAsor and FA. Recent works indicated that interruption of ALDH2 or $\mathrm{ADH} 5$, the major enzymes for FA degradation, may cause the accumulation of DNA damage and related diseases such as bone marrow failure, as well as dysfunction of liver and kidney ${ }^{4-6}$. We utilized inhibitors to simulate the ALDH2/ADH5 deficient condition to investigate the endogenous FA fluctuations ${ }^{40,41}$. When the FAsor-transfected HeLa cells were treated with daidzin (ALDH2 inhibitor), N6022 (ADH5 inhibitor) alone or together, and vehicle control respectively, cells with different treatment of inhibitors all showed increased $F_{488 \mathrm{~nm}} / F_{405 \mathrm{~nm}}$ ratio than that of vehicle-treated cells (Fig. 4c, d). Moreover, all of inhibitorstreated cells showed higher response upon subsequent incubation with $0.1 \mathrm{mM}$ FA (Fig. 4c, d). The higher response upon 
a

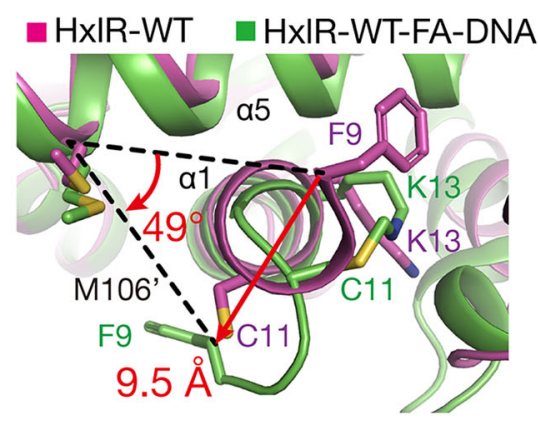

b

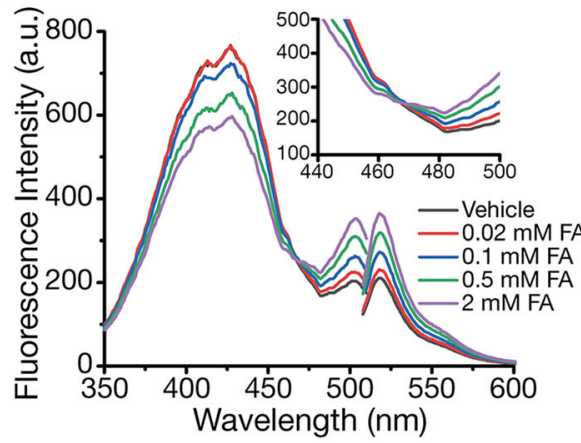

FAsor: a genetically encoded single-chain Formaldehyde (FA) sensor

HxIR monomer $>\quad$ cPYFP $>$ HxIR monomer

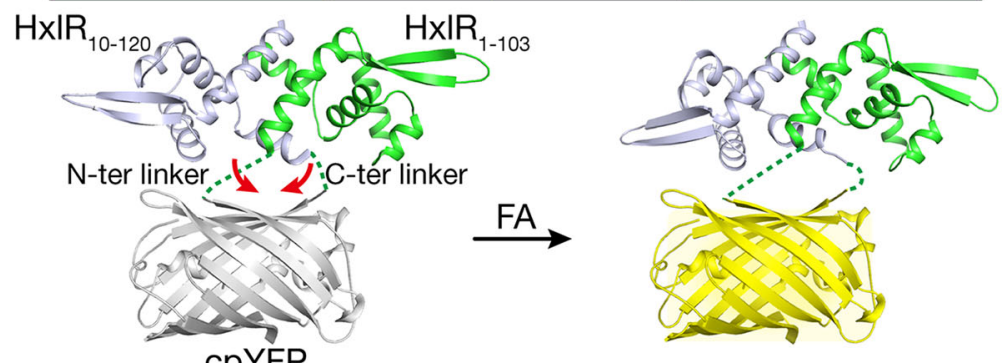

C

d
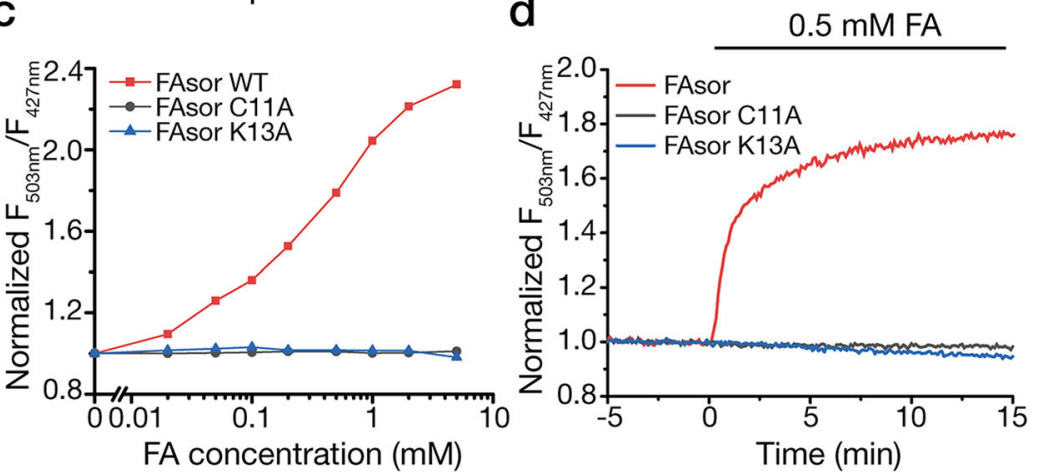

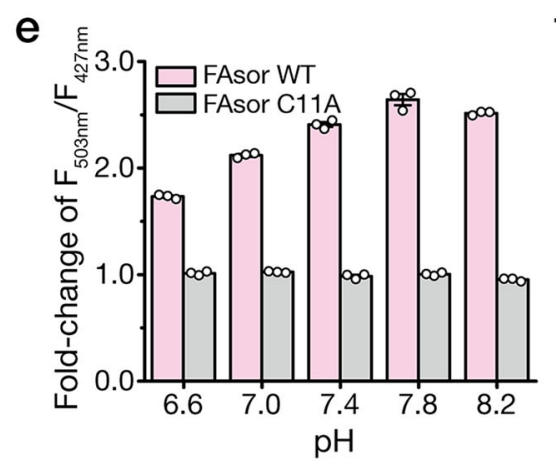

f

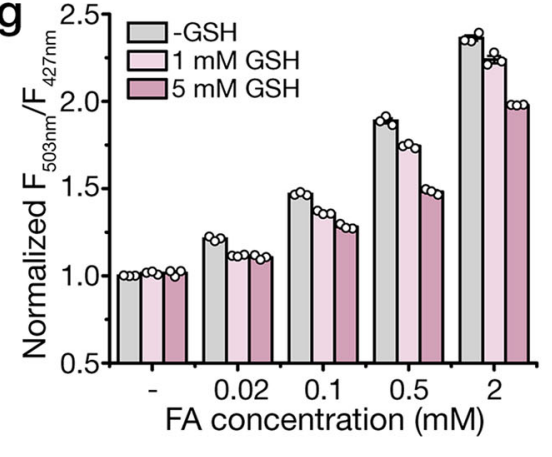

Fig. 3 Development and characterization of the HxIR-based FA sensors (FAsors). a Structure-guided design of the genetically encoded FA sensorFAsor for detecting conformational change in HxIR protein. FA-triggered Cys11-Lys13 crosslinking induces large translocation of residue Phe9 on the same helix ( $9.5 \AA$ and turned 49 degree to Met106' on helix $\alpha 5$ ). By fusion of a cpFP between two subunits in HxIR dimer protein, the resulting single-chain sensor (HxIR-cpYFP-HxIR) can transduce the FA-induced conformational change into the fluorescence signal change. Red arrows indicate distance of Phe9 translocation during conformational change. $\mathbf{b}$ Fluorescence response of FAsor in the presence of $F A$. Excitation and emission spectra of FAsor in the absence and presence of different concentrations of FA are shown. FAsor exhibited a ratiometric property in its excitation spectrum, with its fluorescence increased above $467 \mathrm{~nm}$ and decreased below $467 \mathrm{~nm}$ upon FA treatment. c The ratiometric change of FAsor in response to serial concentrations of FA ranging from 0 to $5 \mathrm{mM}$ in $30 \mathrm{~min}$. The ratio is calculated as the fluorescence intensity of 503-nm excitation peak divided by that of the $427-\mathrm{nm}$ excitation peak, with the emission filter fixed at $516 \mathrm{~nm}$. FAsor-WT responded to FA quantitatively while FAsor-C11A and FAsor-K13A mutants remained unchanged. d Kinetics measurement of FAsor in response to FA. $0.5 \mathrm{mM} F A$ was added to $10 \mu \mathrm{M}$ FAsor. Fluorescence ratio of FAsor-WT increase rapidly upon addition of FA, while FAsor-C11A and FAsor-K13A mutants remain stable. e Response of FAsor-WT/C11A to FA at different pH. The columns indicate fluorescence change of FAsor-WT/C11A before and after addition of $2 \mathrm{mM} \mathrm{FA}$ in buffers with different $\mathrm{pH}$. FAsor-WT is able to sense FA between pH 6.6-8.2 with the FAsor-C11A control probe showing negligible responses in the same $\mathrm{pH}$ range. $\mathbf{f}$ Fluorescence response of FAsor to biologically relevant carbonyl metabolites and hydrogen peroxide. Data shown are for $0.5 \mathrm{mM}$ of all species. $\mathbf{g}$ The effects of GSH on FAsor. The fluorescent response of FAsor to FA retains up to $\sim 80 \%$ in the presence of $5 \mathrm{mM} \mathrm{GSH}$. Data in $\mathbf{e}-\mathbf{g}$ are shown in mean \pm SEM for three measurements.

subsequent incubation of FA had also been verified by flow cytometry (Fig. 4e, Supplementary Fig. 6a). This result confirmed that the deficiency of FA metabolism in ALDH2/ADH5 inhibited cells disrupted FA homeostasis and caused cellular FA accumulation, which may lead to cell damaging effects in related diseases. In addition, a recent report showed that inhibition of GSH synthesis with L-BSO (L-Buthionine-Sulfoximine, an inhibitor of $\gamma$-glutamylcysteine synthetase) promoted the toxicity of FA to cells, especially in those with ADH5 knockout ${ }^{42}$. We thus treated
HeLa cells with L-BSO and N6022 alone or together, and vehicle control, respectively. L-BSO induced similar FAsor signal increase compared with N6022 (Supplementary Fig. 6b, c). Combined treatment of L-BSO and N6022 resulted in little increase with no statistical difference (Supplementary Fig. 6b, c), indicating that ADH5 and GSH synthesis might be epistatic for the control of intracellular $\mathrm{FA}^{42}$.

Next, we employed FAsor to visualize endogenous FA dynamics in tetrahydrofolate (THF) metabolism. A recent work 
a

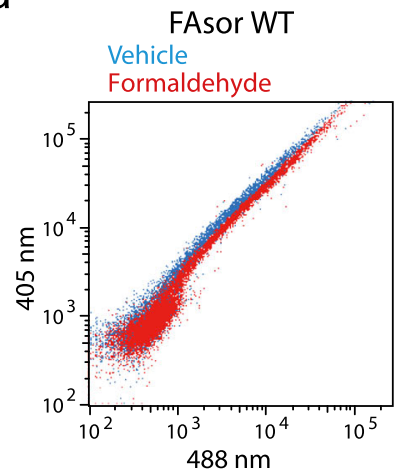

FAsor C11A

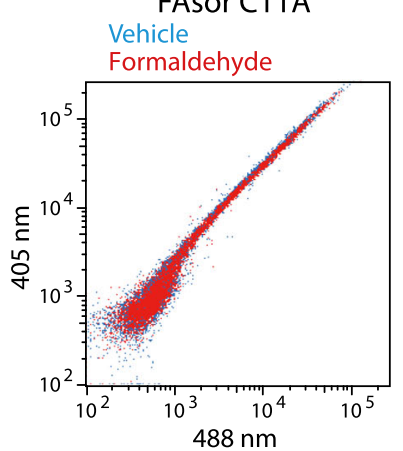

b
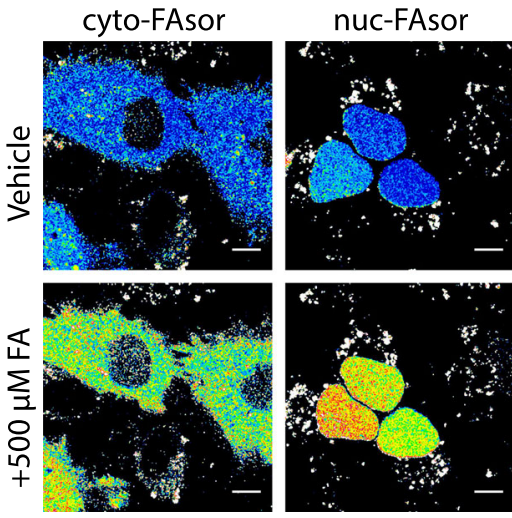

d
C
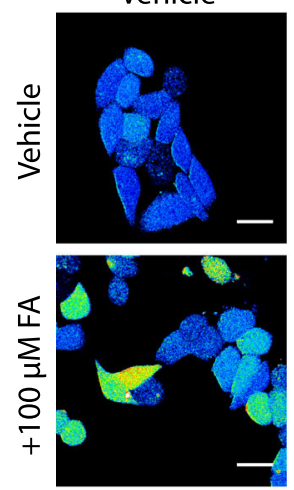

e

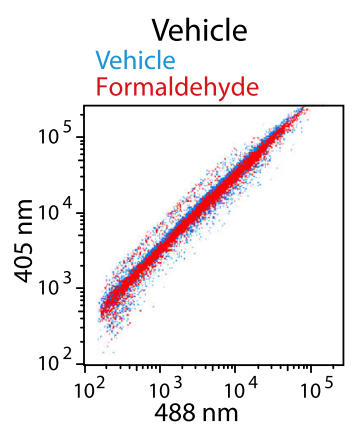

$f$
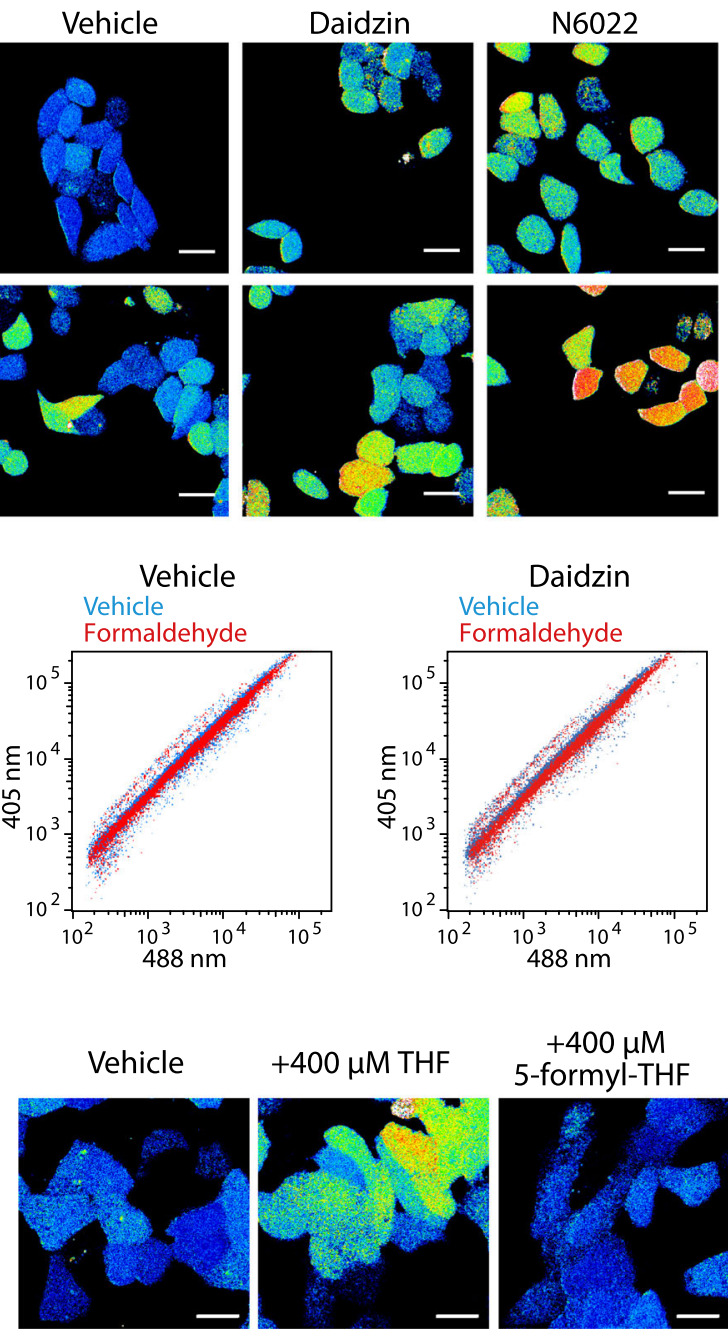

$+400 \mu \mathrm{M}$ 5-formyl-THF

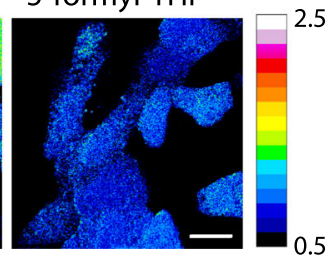

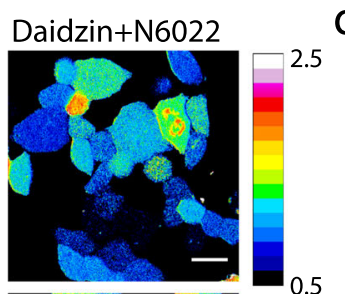
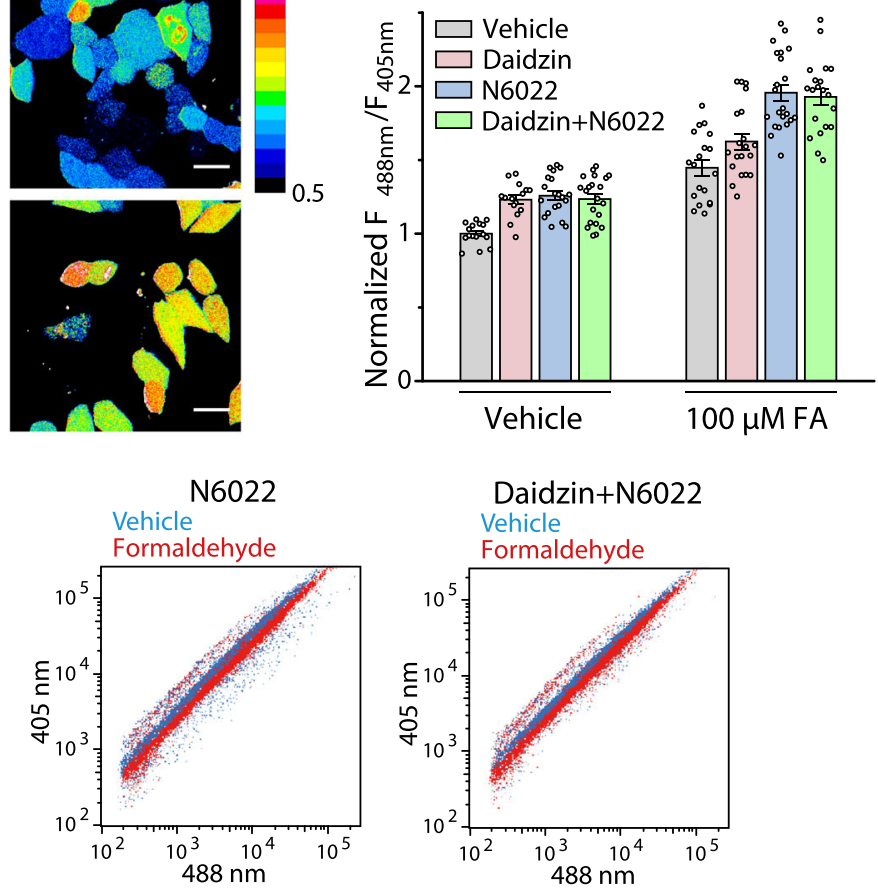

g

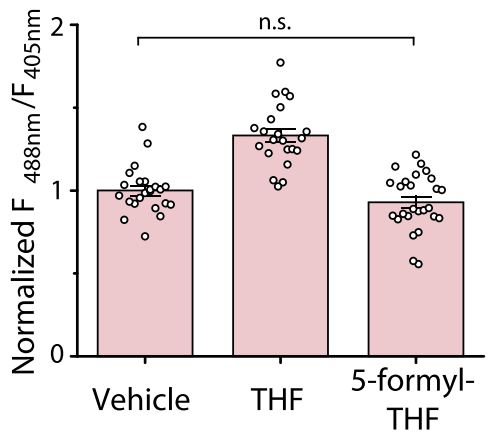

indicated that oxidative THF decomposition can release FA, which may serve as the source of $1 \mathrm{C}$ units and support nucleotide synthesis $^{12}$. We thus utilized FAsor to detect endogenous FA fluctuation upon the addition of THF. A significant increase of $F_{488 \mathrm{~nm}} / F_{405 \mathrm{~nm}}$ ratio was observed on HeLa cells treated with 0.4 $\mathrm{mM}$ THF (Fig. 4f, g). In contrast, treatment of cells with 5formyl-THF, which is resistant to oxidation and unable to release $\mathrm{FA}^{12}$, induced negligible signal changes (Fig. 4f, g). In summary, we have established that FAsors could detect endogenous FA fluctuation and metabolism.
Visualization of FA dynamics in mouse brain tissues by FAsors. Compared with small molecule probes, genetically encoded fluorescent sensors provide the possibility of cell-type-specific expression as well as potential long-term imaging in animals. Cells transfected with FAsor or infected with Adeno-associated virus (AAV) containing FAsor could respond to FA with fluorescence changes under confocal or two-photon microscope, while cells expressing FAsor-C11A did not respond to FA (Supplementary Fig. 7a-d). To test the applicability of FAsor in detecting FA dynamics in a physiological relevant system, we injected AAV 
Fig. 4 Spatial and temporal visualization of FA by FAsors in living cells. a FACS measurements of FAsor's $F_{488 \mathrm{~nm}} / F_{405 \mathrm{~nm}}$ ratio in response to exogenous FA in HEK293T cells. Cells are incubated at $37^{\circ} \mathrm{C}$ with $0.5 \mathrm{mM} \mathrm{FA}$ for $1 \mathrm{~h}$. FA induced the increase of $F_{488 \mathrm{~nm}} / F_{405 \mathrm{~nm}}$ ratio for cells expressing FAsor-WT but not cells expressing FAsor-C11A. b Fluorescence imaging of subcellular-targeted FAsor upon the treatment of FA. HeLa cells were treated with 0.5 mM FA for 30 min. FA levels in cytosol, nucleus or mitochondria all undergo changes in response to exogenously added FA. Scale bars, $10 \mu \mathrm{m}$. c, $\mathbf{d}$ Fluorescence imaging of FAsor without subcellular-targeting sequence in response to endogenous and exogenous FA in different states of FA metabolism. HeLa cells were treated with or without the inhibitors daidzin and/or N6022 for FA-degrading enzymes for $3 \mathrm{~h}$, followed with incubation with or without $0.1 \mathrm{mM} F A$ for $1 \mathrm{~h}$. The enhanced $F_{488 \mathrm{~nm}} / F_{405 \mathrm{~nm}}$ ratio on inhibitor-treated cells indicates higher intracellular $F A$ level due to the suppressed FA degradation. $\mathbf{d}$ is the group summary of $F_{488 \mathrm{~nm}} / F_{405 \mathrm{~nm}}$ under different conditions. For groups from left to right, $n=16,15,20,21,19,20,22$, and 20 cells, respectively. Scale bars, $25 \mu \mathrm{m}$. e FACS measurements of FAsor's $F_{488 \mathrm{~nm}} / F_{405 \mathrm{~nm}}$ ratio in response to FA in different states of FA metabolism. HEK293T cells are incubated with different inhibitors ( $10 \mu \mathrm{M}$ each) at $37^{\circ} \mathrm{C}$ for $3 \mathrm{~h}$, followed with or without addition of $0.4 \mathrm{mM} \mathrm{FA}$ for another $1 \mathrm{~h}$. $\mathbf{f}, \mathbf{g}$ Fluorescence imaging of the FA generation via THF metabolism by FAsor without subcellular-targeting sequence. HeLa cells were treated with $0.4 \mathrm{mM}$ THF or 5-formyl-THF for 30 min. The increased $F_{488 \mathrm{~nm}} / F_{405 \mathrm{~nm}}$ ratio indicated endogenously generated FA molecules. $\mathbf{g}$ is the group summary of $F_{488 \mathrm{~nm}} / F_{405 \mathrm{~nm}}$ under different conditions. For groups from left to right, $n=23,22$, and 26 cells, respectively. Unpaired two-sided student's $t$-test was performed. n.s. no significance $(P=0.12)$. Scale bars, $20 \mu \mathrm{m}$. In $\mathbf{b}, \mathbf{c}, \mathbf{f}$, images were pseudocolored with normalized $F_{488 \mathrm{~nm}} / F_{405 \mathrm{~nm}}$ ratio. Data in $\mathbf{d}, \mathbf{g}$ are shown in mean $\pm \mathrm{SEM}$.

containing either FAsor or FAsor-C11A in the hippocampus dentate gyrus region of mice, and prepared acute brain slices 3 weeks after in vivo viral expression (Fig. 5a). Both FAsor and FAsor-C11A showed robust fluorescent signal in the pyramidal neurons of the dentate gyrus under two-photon microscope (Fig. 5b), indicating their successful expression.

Next, we treated the sensor-expressing acute brain slices with exogenous perfusion of FA or acetaldehyde (ACA). The application of $0.5 \mathrm{mM}$ FA elicited fluorescence signal decrease in FAsor-expressing neurons at single cell level, with the averaged $\Delta \mathrm{F} / \mathrm{F}$ around $-30 \%$ (Fig. $5 \mathrm{c}-\mathrm{e}$, red), while ACA did not cause detectable fluorescence changes (Fig. $5 \mathrm{c}-\mathrm{e}$, black), ruling out the possibility of fluorescence change caused by environmental changes. Furthermore, in neurons expressing FAsor-C11A, application of either ACA or FA did not cause observable fluorescence changes (Fig. 5f-h), consistent with the result at protein level (Fig. 3c). Altogether, these results indicated that FAsor could specifically detect the dynamics of FA in mouse brain tissues.

\section{Discussion}

FA has long been considered as a detrimental molecule, with the potential physiological role remaining controversial ${ }^{1,5,6,12}$. This is largely due to the lack of direct evidence supporting the beneficial effects of FA on a given biomolecule or cellular machinery as opposed to the nonspecific damaging effects. Our study on the FA-sensing transcription factor HxlR revealed that FA induced a specific intrahelical crosslinking reaction between residues Cys11 and Lys13. The resulting methylene bridge (Cys- $\mathrm{CH}_{2}$-Lys) on HxlR's helix al caused unwinding and rotation of its N-terminus ("N-terminal helix-flipping") that allosterically rotated its DNAbinding domain with enhanced DNA affinity and transcriptional activation. This FA-triggered covalent Cys-Lys crosslinking represents a modification on proteins with a gain-of-function effect. This may shed light on how cells manage to sense highly reactive species such as FA. Furthermore, in addition to FA, other RCS with multivalent reactive groups may also influence protein conformation and functions through intramolecular or intermolecular crosslinking. All these findings represent a general mechanism how metabolic pathways interact with stress-responsive signaling through electrophiles, especially in a gain-of-function crosslinking manner ${ }^{43-46}$, which merits further investigation by quantitative proteomics in the future.

Recent works have revealed a RcnR/CsoR family derepressor, E. coli FrmR, that responded to FA via crosslinking between cysteine and the $\mathrm{N}$-terminal proline ${ }^{29}$. The secondary amine group from the proline residue is essential, as mutating it to primary amine (e.g., Pro to Ser/Ala mutation) abolished FA sensing by FrmR in vivo ${ }^{29,30}$. In contrast, HxlR represented an example in which protein may also utilize the side-chain of primary amine for FA sensing. The reason why HxlR can utilize primary amine (sidechain of Lys13) to sense FA is yet to be investigated. Despite that the FA-reacting residues are different, the similar crosslinking reactions between the thiol group and amine in HxlR and FrmR exhibited how FA regulatory proteins specifically distinguish this signal from other thiol-reactive species such as ROS. Another interesting question is whether the FAinduced crosslinking on regulator proteins, e.g., HxlR/FrmR, is reversible. Recent works indicated that timonacic, the product of cysteine crosslinked by FA, was able to slowly convert back to formaldehyde and cysteine ${ }^{47}$. The authors incubated ${ }^{12} \mathrm{C}$ timonacic with ${ }^{13} \mathrm{C}-\mathrm{FA}$ in $\mathrm{PBS}$ solution $(\mathrm{pH} 7.4)$ and found the sample switched to almost $100 \%{ }^{13} \mathrm{C}$-timonacic after $12 \mathrm{~h}^{47}$. As FA-crosslinking resulted a five-member-ring in timonacic, which is highly stable and not existed in the proteins, we hypothesized that the FA-induced crosslinking on regulator proteins can be reversed in a faster kinetics. The intracellular thiols may further promote the reverse reaction of FA-induced crosslinking. Additionally, the degradation of the FA-crosslinked proteins in cells, such as through the Clp-dependent proteolysis, is also a potential mechanism in physiological environment.

Inspired by the unique FA-sensing mechanism of HxlR, we developed genetically encoded sensors-FAsor and FAsorRed that were able to transduce the FA-induced protein conformational change to a fluorescent signal. This allowed us to detect and visualize FA dynamics in various physiological contexts with high spatial-temporal resolution. Recently, a number of groups made developments of small molecule FA sensors, which can be classified as 2-aza-Cope-based sensors and formimine/aminal-based sensors by their sensing mechanism ${ }^{21,22}$. 2-aza-Cope-based sensors have high sensitivity and specificity for FA, but with low reaction kinetics (may take hours to get reasonable signal change) and are irreversible ${ }^{17,18}$. Formimine- and aminal-based sensors usually react rapidly and reversible with FA (the signal changes within $10 \mathrm{sec}$ to a few minutes), while they also respond to simple aldehydes such as acetaldehyde ${ }^{19,20}$. The reaction kinetics, sensitivity and specificity of FAsor, which can sense $20 \mu \mathrm{M}$ FA within $5 \mathrm{~min}$, are more similar with the formimine/aminal-based small molecular sensors. As a ratiometric sensor, FAsor have lower turn-on ratio compared to the most intensiometric small molecular FA sensors (some of which may have 900-fold turn-on upon reaction with FA). Instead, FAsor avoided from suffering the signal fluctuation induced by varied quantity of the sensor. FAsor has the dual specificity towards FA and MG as HxlR is able to respond to both molecules. As MG has a much lower endogenous concentration than $\mathrm{FA}^{39}$, in most cases the main signal change of FAsor in vivo would be induced by FA. The application of FAsor would be most appropriate when the FA source is clear, or 
a

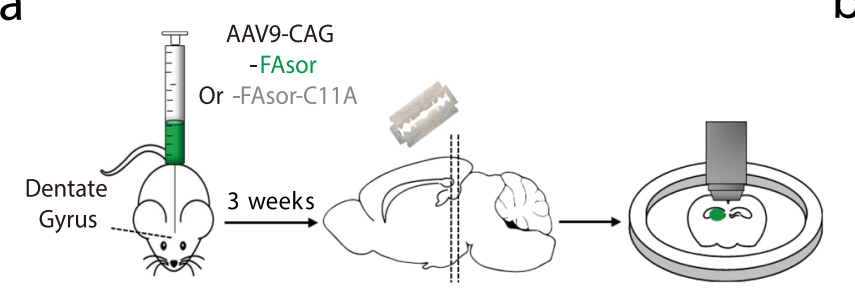

Virus injection Acute slice preparation

C

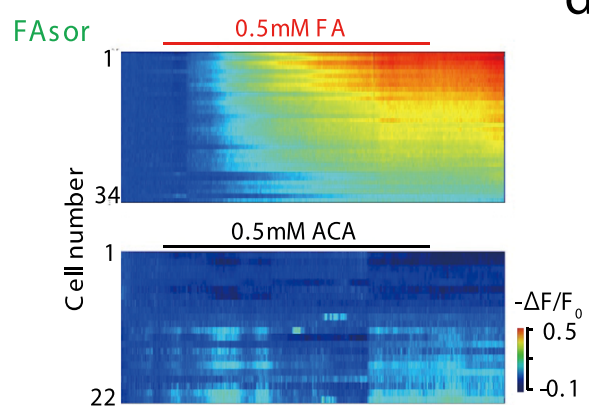

f

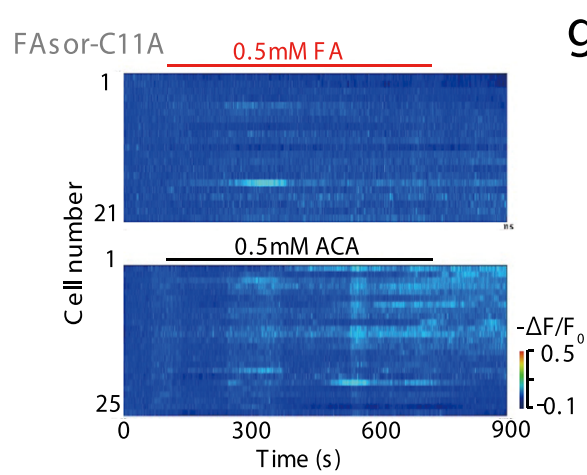

b

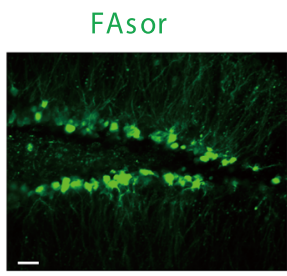

Two-photon imaging

d

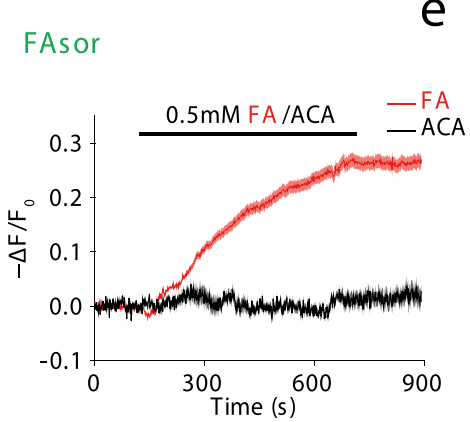

h

\section{FAsor-C11A}

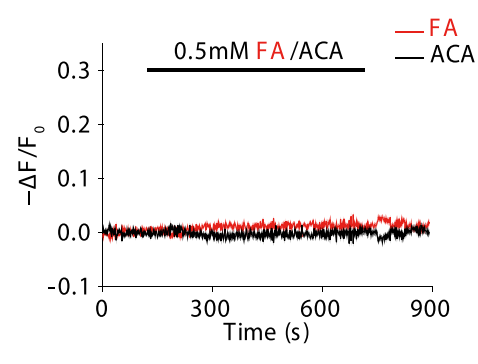

FAsor-C11A

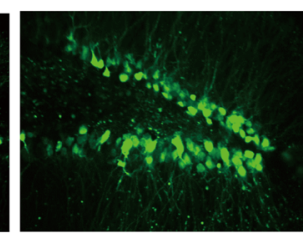

e

FAsor

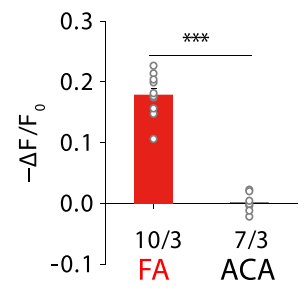

FAsor-C11A

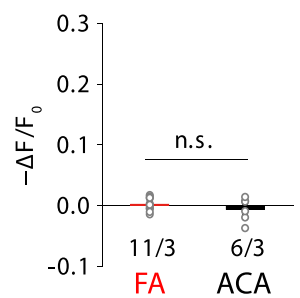

Fig. 5 Visualization of FA in mouse acute brain slices by two-photon imaging of FAsors. a Schematic illustration of the workflow. AAVs containing FAsor or FAsor-C11A were injected into the dentate gyrus of mice, and acute brain slices were prepared 3 weeks after viral expression, which were placed under two-photon microscope for imaging. $\mathbf{b}$ Expression of FAsor and FAsor-C11A in pyramidal neurons of the dentate gyrus in acute brain slices. The two-photon laser was set at $880 \mathrm{~nm}$ for excitation. Scale bars: $20 \mu \mathrm{m}$. c-e The fluorescence responses of FAsor-expressing neurons to exogenous perfusion of $0.5 \mathrm{mM}$ formaldehyde (FA) (upper, red), or $0.5 \mathrm{mM}$ acetaldehyde (ACA, lower, black) as a control. The responses of individual cells in one single slice were plotted in pseudo-color in c, and their averaged time-dependent traces were shown in $\mathbf{d}$. The group data of responses in multiple slices of different animals were summarized in e with data shown in mean \pm SEM. Unpaired two-sided student's $t$-test was performed. $n=10$ slices from 3 mice and 7 slices from 3 mice, for FA and ACA, respectively. ${ }^{\star \star \star} P<0.001\left(P=3.19 \times 10^{-7}\right)$. f-h Similar as $\mathbf{c}-\mathbf{e}$, except the fluorescence responses of FAsor-C11A-expressing neurons were summarized and plotted. Data are shown in mean \pm SEM in $\mathbf{h}$. Unpaired two-sided student's $t$-test was performed. $n=11$ slices from 3 mice and 6 slices from 3 mice, for $\mathrm{FA}$ and ACA, respectively. n.s. no significance $(P=0.35)$.

distinguishing signals from FA and MG is not crucial, such as the preliminary screening whether FA/MG level were enhanced in a disease model or under certain treatment, specific process. Further research on sensing mechanism of HxlR to $\mathrm{MG}$ may lead to the development of MG and FA specific sensors. Notably, another advantage for the genetically encoded, fluorescent protein (FP)based sensors is subcellular targeting. Subcellular-targeted FAsor variants were also developed for simultaneously monitoring FA in different subcellular compartments. Our sensors provide a toolkit that may help to improve our understanding of the potential roles of FA in various cellular processes. Some recent research examined Fanconi anemia patients and found that people holding the ALDH2 dominant-negative variant (ALDH2*2) had the tendency of accelerated progression of bone marrow failure $(\mathrm{BMF})^{48,49}$. Study on Fancd2, Aldh2 and Adh5 knockout mice have indicated the effect of increased endogenous formaldehyde in the process ${ }^{4,5}$. We have confirmed elevated endogenous formaldehyde upon inhibition of ALDH2 with FAsor, and the putative role of endogenous formaldehyde in $\mathrm{ALDH} 2 * 2$ alleles worth further investigation. In particular, the application of FAsors in mouse brain slices may facilitate the study of the underlying connections between FA and neurodegenerative diseases.

\section{Methods}

Primary cultures. Rat cortical neurons were prepared from postnatal 0-day old (P0) Sprague-Dawley rat pups (male and female, random choice; Beijing Vital River). The cortical neurons were dissociated from the dissected rat brains in $0.25 \%$ Trypsin-EDTA (GIBCO), and plated into LabTek 8-well chambered coverglass (NUNC) in neurobasal medium (GIBCO) containing 2\% B-27 supplement (GIBCO), 1\% GlutaMax (GIBCO), and 1\% penicillin-streptomycin (GIBCO). The neurons were cultured at $37^{\circ} \mathrm{C}$ in $5 \% \mathrm{CO}_{2}$.

Bacterial strains. E. coli strains DH5 $\alpha$ and BL21(DE3) were purchased from Tiangen Biotech. Bacillus subtilis strains 168 was purchased from American Type Culture Collection (ATCC). E. coli strains BW25113 was obtained from the National BioResource Project (National Institute of Genetics, Japan).

Cell lines. HEK293T cell line (female, ATCC, CRL-11268) and HeLa cell line (female, ATCC, CCL-2) were purchased from ATCC. The cell lines were 
authenticated by the morphology under microscopy and the growth curve analysis. Cells were maintained in DMEM (GIBCO) media at $37^{\circ} \mathrm{C}$ in $5 \% \mathrm{CO}_{2}$. All cell lines were supplemented with $10 \%$ fetal bovine serum and $1 \%$ penicillin-streptomycin (GIBCO).

Mice. Postnatal 56- to 70-day-old (P56-70) wild-type C57BL/6 mice were used to prepare the acute brain slices (male and female, random choice; Beijing Vital River). All mice were either family-housed or pair-housed in a temperaturecontrolled room (21.5 degree centigrade) with a 12-h/12-h light/dark cycle, with humidity controlled as $55 \%$. All procedures for animal surgery and maintenance were performed using protocols that were approved by the Animal Care \& Use Committees at Peking University, and were performed in accordance with the guidelines established by US National Institutes of Health guidelines.

Plasmids construction. The gene of HxlR (UniProt number: P42406 [https:// www.uniprot.org/uniprot/P42406]) from strains 168 of Bacillus subtilis was subcloned into pET28a between sites NdeI and XhoI with stop codon before Cterminal his-tag. Site-directed mutagenesis was applied to obtain HxlR mutants based on the QuickChange ${ }^{\text {tw }}$ method developed by Stratagene (La Jolla, CA). Oligonucleotides used for the mutagenesis reactions are listed in Supplementary Table 2. The $h x l A B-g f p$ reporter plasmid was constructed by subcloning $h x l A B$ promoter from $B$. subtilis 168 into $\mathrm{pL}(\mathrm{marO})$-GFP plasmid between sites XhoI and KpnI, replacing the original marO promoter ${ }^{50}$. pBAD-HxlR was constructed by replacing the DiZPK-RS and Mb-tRNA-pyl in pSupAR plasmid with the gene of HxlR protein via Gibson Assembly ${ }^{51,52}$. Constructions of HxlR-cpYFP-HxlRexpressing plasmids were achieved via Gibson Assembly and/or QuickChange ${ }^{\mathrm{n}}$ method $^{51}$. For eukaryotic expressing plasmids (pcDNA-based plasmids), a synthesized codon-optimized HxlR gene (by Genewiz, the sequence is listed in Supplementary Table 3) was utilized as template.

Protein expression and purification. For expression of HxlR protein, pET28aHxlR was transformed into E. coli BL21(DE3) strain. Then a single colony was collected and cultured overnight, followed by diluting into $1 \mathrm{~L} \mathrm{LB}$ medium with the ratio of 1:100. For expression of HxlR mutant, the culture was incubated in a shaker at $37^{\circ} \mathrm{C}$ for $3 \mathrm{~h}$ (to $\mathrm{OD}_{600 \mathrm{~nm}} \sim 0.6$ ), and added with $1 \mathrm{mM}$ IPTG (isopropyl $\beta$-D-1-thiogalactopyranoside). The shaker temperature was then adjusted to $30^{\circ} \mathrm{C}$ and the bacteria were harvested after $5 \mathrm{~h}$ incubation. For expression of FAsor, FAsorRed and other HxlR-cpYFP-HxlR protein, the bacteria carrying expressing plasmids based on pET28a was incubated at $25^{\circ} \mathrm{C}$ for $8 \mathrm{~h}$ after addition of IPTG.

Cell pellets were resuspended in lysis buffer $(20 \mathrm{mM}$ HEPES $\mathrm{pH}=7.5,300 \mathrm{mM}$ $\mathrm{NaCl}, 10 \%$ glycerol). After sonicating on ice for $20 \mathrm{~min}$, lysate of bacteria was centrifuged and the supernatant was loaded onto a 5-ml HisTrap HP column (GE Healthcare) before the linear gradient procedure being applied to elute the protein (buffer A: $20 \mathrm{mM}$ HEPES pH =7.5, $300 \mathrm{mM} \mathrm{NaCl}, 10 \%$ glycerol; buffer B: $20 \mathrm{mM}$ HEPES pH $=7.5,300 \mathrm{mM} \mathrm{NaCl}, 500 \mathrm{mM}$ Imidazole, $10 \%$ glycerol). The HxlR components were collected and further concentrated. Overnight thrombin digestion (HxlR protein diluted to $1 \mathrm{mg} / \mathrm{ml}$ in $20 \mathrm{mM}$ Tris- $\mathrm{HCl}, \mathrm{pH}=8.4,150 \mathrm{mM} \mathrm{NaCl}$, $2.5 \mathrm{mM} \mathrm{CaCl}_{2}$ with $1 \mathrm{U} / \mathrm{ml}$ thrombin added) at $16^{\circ} \mathrm{C}$ was performed to remove the $\mathrm{N}$-terminal His-tag. After digestion, the protein sample was treated with $10 \mathrm{mM}$ DTT for $15 \mathrm{~min}$ to reduce potential oxidation and next purified by size exclusion chromatography with HiLoad 16/60 Superdex 200 (GE Healthcare) equilibrated with $20 \mathrm{mM}$ HEPES $\mathrm{pH}=7.5,300 \mathrm{mM} \mathrm{NaCl}$. All the $\mathrm{pH}$ 's of the buffers were measured at room temperature. The purifications were performed at $4{ }^{\circ} \mathrm{C}$

Crystallization, X-ray data collection, and structure determination. All the crystallization experiments in this study were performed with sitting-drop vapor diffusion at $20^{\circ} \mathrm{C}$. Crystals of HxlR-WT and HxlR-K13A were screened with kits from Hampton Research (Index, SaltRx, Crystal Screen, PEGRx, PEG/Ion Screen). $1.5 \mu \mathrm{l} \mathrm{HxlR}$ protein solution $(\sim 5 \mathrm{mg} / \mathrm{mL}$ in $20 \mathrm{mM}$ HEPES $\mathrm{pH}=7.5,300 \mathrm{mM}$ $\mathrm{NaCl})$ and $1.5 \mu \mathrm{l}$ reservoir solution were mixed. Single crystals could be observed after $24 \mathrm{~h}$. For crystallization of HxlR-WT-FA-DNA complex, HxlR-WT $(\sim 5 \mathrm{mg} /$ $\mathrm{mL}$ in $20 \mathrm{mM}$ HEPES $\mathrm{pH}=7.5,150 \mathrm{mM} \mathrm{NaCl}$ ) was treated with $1 \mathrm{mM} \mathrm{FA}$ for $1 \mathrm{~h}$ at $37^{\circ} \mathrm{C}$. This solution was next mixed with the same volume of double-stranded DNA solution (final Protein:DNA ratio $=1: 1.5$ or $1: 2$ ) for at least $30 \mathrm{~min}$ at $4{ }^{\circ} \mathrm{C}$. The final mixtures were screened with reservoir solutions containing $0-100 \mathrm{mM}$ $\mathrm{NaCl}, 50-200 \mathrm{mM} \mathrm{MgCl}_{2}, 100 \mathrm{mM}$ buffering reagent (Bis-tris, MES or sodium cacodylate) at various $\mathrm{pH}(5.0-7.0)$, and different concentrations of PEGs (PEG $1000,2000,3350,4000,6000,8000$, PEG MME 550, 5000) (w/v or v/v 10-40\%). The sequence of DNA generating crystals of HxlR-WT-FA-DNA complex that has the best diffraction was 5'- CAG TAT CCT CGA GGA TAC TG -3'. The growing conditions for crystals with the best diffraction quality are $0.1 \mathrm{M}$ sodium acetate trihydrate $\mathrm{pH}=4.6,1.5 \mathrm{M}$ ammonium chloride, $10 \mathrm{mM}$ DTT for HxlR-WT, $0.2 \mathrm{M}$ DL-Malic acid pH 7.0, 20\% w/v PEG 3350 for HxlR-K13A and $0.1 \mathrm{MES} \mathrm{pH}=6.4$, $50 \mathrm{mM} \mathrm{MgCl} 2,25 \%$ v/v PEG MME 550 for HxlR-WT-FA-DNA.

Crystals were washed with cryoprotectant freshly made by mixing reservoir solution with $90 \%$ glycerol in 7:2 ratio, and then flash frozen in liquid nitrogen prior to X-ray diffraction. Diffraction datasets of HxlR-WT and HxlR-WT-FADNA were collected by beamlines of BL17U1 at Shanghai Synchrotron Radiation Facility $(\mathrm{SSRF})^{53}$ with wavelength at $0.9792 \AA$ for HxlR-WT and $0.9793 \AA$ for
HxlR-WT-FA-DNA, respectively. Diffraction datasets of HxlR-K13A were collected by beamlines of BL19U1 at SSRF with wavelength at $0.9785 \AA$ A Diffraction datasets were collected with Blu-Ice software. The collected datasets were processed with HKL2000 or HKL3000 54,55 , and structures were solved via molecular replacement with programs Phaser $^{56}$ in CCP4 package ${ }^{57}$. Structures of HypR (Protein Data Bank code 4A5N [https://www.rcsb.org/structure/4A5N] ${ }^{32}$ and QsrR-DNA complex (Protein Data Bank code 4HQE [https://www.rcsb.org/ structure/4HQE] $)^{33}$ are used as search models for HxlR protein and HxlR-WT-FADNA complex, respectively. Refinement was performed by programs REFMAC5 $5^{58}$ in CCP4 package ${ }^{57}$, Phenix ${ }^{59}$ and $\mathrm{COOT}^{60}$. The Ramachandran plot given by MolProbity ${ }^{61}$ in Phenix ${ }^{59}$ showed that in every structure, no residue was in the disallowed region. Protein sequence alignment was performed by ClustalO ${ }^{62}$. PyMOL was used to generate all the figures. Summary of the data statistics is listed in Supplementary Table 1.

Mass spectrometry and data analysis. For FT-MS analysis, purified HxlR (at $100 \mu \mathrm{M}$ ) was incubated with $1 \mathrm{mM}$ FA in $20 \mathrm{mM}$ HEPES $\mathrm{pH}=7.5,300 \mathrm{mM}$ $\mathrm{NaCl}$ for $1 \mathrm{~h}$ at $37^{\circ} \mathrm{C}$. The protein sample was next desalted by Bio-Rad Micro BioSpin 6 column and subjected to mass spectrometric analysis by Bruker Solarix XR system. For MS-MS analysis of HxlR protein, $0.6 \mathrm{mM}$ FA was added to the medium of $E$. coli BL21(DE3) expressing HxlR at $1 \mathrm{~h}$ before harvest. The FA-treated cells were then collected and HxlR protein was purified with HisTrap HP column as described above. The purified protein was then digested with trypsin and the resulted peptide sample was next desalted with Thermo Scientific Pierce C18 tips and subjected to Thermo Scientific Q Exactive Plus Orbitrap LC-MS/MS system, with the data collected by Xcalibur software. For LC-MS analysis of synthetic 7amino acid peptides, the peptides were incubated with $1 \mathrm{mM} \mathrm{FA}$ in water for $1 \mathrm{~h}$ at $37^{\circ} \mathrm{C}$. The peptide samples were then analyzed with a Waters ACQUITY UPLC IClass SQD 2 MS spectrometer with electrospray ionization (ESI). Data collection was achieved by Masslynx software.

Electrophoretic mobility shift assays (EMSA). EMSA was performed as described previously ${ }^{50}$. Briefly, $100 \mu \mathrm{M}$ HxlR proteins (HxlR-WT, HxlR-C11A and HxlR-K13A) were incubated with or without $1 \mathrm{mM}$ FA in $20 \mathrm{mM}$ HEPES $\mathrm{pH}=$ $7.5,300 \mathrm{mM} \mathrm{NaCl}$ for $1-2 \mathrm{~h}$ at $37^{\circ} \mathrm{C}$. After incubation, the proteins were diluted to a final volume of $20 \mu \mathrm{l}$ containing $50 \mathrm{nM}$ annealed double-stranded DNA in binding buffer (20 mM HEPES pH 7.0, $50 \mathrm{mM} \mathrm{KCl}, 5 \mathrm{mM} \mathrm{MgCl}_{2}$, and $10 \%$ glycerol). Sequences of oligonucleotides used for the annealing process was listed in Supplementary Table 2 .

Flow cytometry analysis of HxIR-mediated transcription E. coli. An overnight $E$. coli BW25113 culture harboring both the hxlAB-gfp reporter plasmid and the pBAD-HxlR plasmid was inoculated (1:100) in LB medium and grown to an $\mathrm{OD}_{600 \mathrm{~nm}}$ of 0.6 . L-Arabinose with $4 \mathrm{mM}(\sim 0.06 \% \mathrm{w} / \mathrm{v})$ in final concentration was added to induce expression of HxlR protein. After $1 \mathrm{~h}$ induction, bacterial cells were next treated with or without $600 \mu \mathrm{M} \mathrm{FA}, 40$ min before being analyzed by flow cytometry. Fluorescence of the GFP channel (EX $488 \mathrm{~nm}$, EM 530/30BP) were measured with the BD LSRFortessa flow cytometer (BD Biosciences). To assess the effect of FA on viability of bacteria, E. coli BW25113 grown to an $\mathrm{OD}_{600 \mathrm{~nm}}$ of 0.6 were allow to grow at $37^{\circ} \mathrm{C}$ for $1 \mathrm{~h}$, and followed by treating with/without $600 \mu \mathrm{M}$ FA for $40 \mathrm{~min}$. Next, 10 folds of serial dilutions of the bacteria with/without FAtreatment analysis were plated for colony counts.

Expression of HYH-5 in cultured cells. HEK293T cells were cultured in 24-well culture plates (Corning) and HeLa cells were cultured in LabTek 8-well chambered coverglass (NUNC), and grown to a confluency of $\sim 50 \%$ for transfection. Lipofectamine 2000 (Invitrogen) were used for transfection of HEK293T cells and XTremeGene HP (Roche) for transfection of HeLa cells. Transfection was performed according to the manufactures' protocols. Imaging was performed $18-24 \mathrm{~h}$ after transfection.

The cultured neurons were infected $\sim 10$ days later after dissection using AAV. The infection was performed by adding $1 \mu \mathrm{L}$ AAV9-CAG-FAsor/FAsor-C11A into the medium in one well of 24 -well plate. Imaging was performed $48-72 \mathrm{~h}$ after infection.

In vitro measurement of fluorescence. Generally, $10 \mu \mathrm{M}$ purified FAsor/FAsorRed protein was incubated with different concentrations of FA or other compounds for $5 \mathrm{~min}$ before recording the fluorescent signal. All of the experiments were performed in $20 \mathrm{mM} \mathrm{HEPES} \mathrm{pH}=7.5,300 \mathrm{mM} \mathrm{NaCl}$ at room temperature on the Cary Eclipse Fluorescence Spectrophotometer (Agilent Technologies, U.S.A.). For excitation spectra of FAsor/FAsorRed protein, intensity of fluorescence emission at $516 \mathrm{~nm}$ or $594 \mathrm{~nm}$ was measured respectively if not specified. And for emission spectra of FAsor/FAsorRed protein, the excitation filter is fixed at $503 \mathrm{~nm}$ or $569 \mathrm{~nm}$, respectively. In the kinetics study, fluorescence emission intensities at $516 \mathrm{~nm}$ of FAsor excited at $427 \mathrm{~nm}$ and $503 \mathrm{~nm}$ were recorded every $6 \mathrm{~s}$, respectively. Cary Eclipse Software was used for data collection. 
Flow cytometry analysis of cultured cells. For flow cytometry, HEK293T cells transfected with FAsor in a 24-well culture plate (Corning) were changed to fresh DMEM with different reagents. After incubation the cells were trypsinized and resuspended into $0.5 \mathrm{~mL}$ PBS. Fluorescence of the AmCyan channel (EX $405 \mathrm{~nm}$, EM 525/50BP after 505LP) and FITC channel (EX $488 \mathrm{~nm}$, EM 530/30BP) were measured with the BD LSRFortessa flow cytometer (BD Biosciences).

Fluorescence imaging in cultured cells. Chambers with HeLa cells were changed to fresh DMEM without phenol red, and subjected to confocal imaging with a LSM700 laser scanning confocal microscopy (ZEISS). Cells expressing FAsor were imaged with both the $405 \mathrm{~nm}$ and $488 \mathrm{~nm}$ excitation, while cells expressing FAsorRed were imaged with the $555 \mathrm{~nm}$ excitation. Live cell time lapse imaging was performed in an incubator maintained at $37^{\circ} \mathrm{C}$ with $5 \% \mathrm{CO}_{2}$. For imaging of subcellular-targeting FAsor constructs, Hoechst33342 or MitoTracker DeepRed (ThermoFisher) was stained before imaging according to the manufactures' protocols. Imaging files were collected via ZEN software and processed with Image $1.52 \mathrm{a}(\mathrm{NIH})$ to measure fluorescence intensity and to generate the ratiometric pseudo-color images.

Fluorescence imaging in brain slices. Brain slice imaging was performed as described previously ${ }^{25}$. In brief, the animals were anesthetized with Avertin, and acute brain slices containing the hippocampus region were prepared in cold slicing buffer containing: $110 \mathrm{mM}$ choline-Cl, $2.5 \mathrm{mM} \mathrm{KCl}, 1.25 \mathrm{mM} \mathrm{NaH}_{2} \mathrm{PO}_{4}, 25 \mathrm{mM}$ $\mathrm{NaHCO}_{3}, 7 \mathrm{mM} \mathrm{MgCl}_{2}, 25 \mathrm{mM}$ glucose, and $2 \mathrm{mM} \mathrm{CaCl}_{2}$. Slices were allowed to recover at $35^{\circ} \mathrm{C}$ in oxygenated Ringers solution containing: $125 \mathrm{mM} \mathrm{NaCl}, 2.5 \mathrm{mM}$ $\mathrm{KCl}, 1.25 \mathrm{mM} \mathrm{NaH}_{2} \mathrm{PO}_{4}, 25 \mathrm{mM} \mathrm{NaHCO}_{3}, 1.3 \mathrm{mM} \mathrm{MgCl}_{2}, 25 \mathrm{mM}$ glucose, and 2 $\mathrm{mM} \mathrm{CaCl}_{2}$ for at least $40 \mathrm{~min}$ before experiments. An Olympus FV1000MPE twophoton microscope equipped with a $\times 40 / 0.80 \mathrm{NA}$ water-immersion objective and a mode-locked Mai Tai Ti:Sapphire laser (Spectra-Physics) were used for imaging the slices. The two-photon excitation wavelength was set as $880 \mathrm{~nm}$, except where indicated otherwise. Formaldehyde or acetaldehyde were added to the physiological solution and perfused into the chamber with slices during imaging. Imaging files were processed with ImageJ $1.52 \mathrm{a}(\mathrm{NIH})$ to measure fluorescence intensity and to generate the ratiometric pseudo-color images. Intensity traces were generated by Origin 2018 and pseudo-color heatmap images were generated by custom-written MATLAB programs.

Statistics and reproducibility. Unless otherwise indicated, data are shown as mean and standard error of mean (SEM), and error bars in figures represent SEM. Unpaired two-sided student's $t$-test was used to determine the $P$ value and no adjustments were made for multiple comparisons in all the statistical test. For EMSA and fluorescence imaging experiments, similar results were obtained from three independent experiments.

Reporting summary. Further information on research design is available in the Nature Research Reporting Summary linked to this article.

\section{Data availability}

Atomic coordinates and structure factors for the crystal structures have been deposited with accession codes PDB ID 7BZD [https://doi.org/10.2210/pdb7bzd/pdb] for HxlRWT, 7BZE [https://doi.org/10.2210/pdb7bze/pdb] for HxlR-K13A and 7BZG [https://doi. org/10.2210/pdb7bzg/pdb] for HxlR-WT-FA-DNA, respectively. The PDB accession code $4 \mathrm{~A} 5 \mathrm{~N}$ corresponding to the HypR protein and PDB accession code $4 \mathrm{HQE}$ corresponding to the QsrR-DNA complex was used in this study. The UniProt accession codes P42406 was also used in this study. Other data are available from the corresponding author upon reasonable request. Source data are provided with this paper.

\section{Code availability}

All codes and programs will be provided upon request.

Received: 5 May 2020; Accepted: 30 November 2020;

Published online: 25 January 2021

\section{References}

1. Kalasz, H. Biological role of formaldehyde, and cycles related to methylation, demethylation, and formaldehyde production. Mini Rev. Med. Chem. 3, 175-192 (2003).

2. Boomsma, F., Hut, H., Bhaggoe, U., van der Houwen, A. \& van den Meiracker, A. Semicarbazide-sensitive amine oxidase (SSAO): from cell to circulation. Med. Sci. Monit. 11, Ra122-Ra126 (2005).

3. Walport, L. J., Hopkinson, R. J. \& Schofield, C. J. Mechanisms of human histone and nucleic acid demethylases. Curr. Opin. Chem. Biol. 16, 525-534 (2012).
4. Garaycoechea, J. I. et al. Genotoxic consequences of endogenous aldehydes on mouse haematopoietic stem cell function. Nature 489, 571-575 (2012).

5. Pontel, L. B. et al. Endogenous formaldehyde is a hematopoietic stem cell genotoxin and metabolic carcinogen. Mol. Cell 60, 177-188 (2015).

6. Tan, S. L. W. et al. A class of environmental and endogenous toxins induces BRCA2 haploinsufficiency and genome instability. Cell 169, 1105-1118 (2017).

7. Heck, H. D., White, E. L. \& Casanovaschmitz, M. Determination of formaldehyde in biological tissues by gas-chromatography mass-spectrometry. Biomed. Mass Spectrom. 9, 347-353 (1982).

8. Heck, H. D. et al. Formaldehyde $(\mathrm{CH} 2 \mathrm{O})$ concentrations in the blood of humans and Fischer-344 rats exposed to $\mathrm{CH} 2 \mathrm{O}$ under controlled conditions. Am. Ind. Hyg. Assoc. J. 46, 1-3 (1985).

9. Tong, Z. Q. et al. Tumor tissue-derived formaldehyde and acidic microenvironment synergistically induce bone cancer pain. PLOS ONE $\mathbf{5}$, e10234 (2010)

10. Rizzi, M., Cravello, B. \& Reno, F. Textile industry manufacturing by-products induce human melanoma cell proliferation via ERK1/2 activation. Cell Proliferat. 47, 578-586 (2014).

11. Rizzi, M., Cravello, B., Tonello, S. \& Reno, F. Formaldehyde solutions in simulated sweat increase human melanoma but not normal human keratinocyte cells proliferation. Toxicol. In Vitro 37, 106-112 (2016).

12. Burgos-Barragan, G. et al. Mammals divert endogenous genotoxic formaldehyde into one-carbon metabolism. Nature 548, 549-554 (2017).

13. Kato, N., Yurimoto, H. \& Thauer, R. K. The physiological role of the ribulose monophosphate pathway in bacteria and archaea. Biosci. Biotechnol. Biochem. 70, 10-21 (2006).

14. Sanghani, P. C. et al. Kinetic mechanism of human glutathione-dependent formaldehyde dehydrogenase. Biochemistry 39, 10720-10729 (2000).

15. Teng, S. et al. The formaldehyde metabolic detoxification enzyme systems and molecular cytotoxic mechanism in isolated rat hepatocytes. Chem. Biol. Interact. 130, 285-296 (2001).

16. Wang, R. S., Nakajima, T., Kawamoto, T. \& Honma, T. Effects of aldehyde dehydrogenase-2 genetic polymorphisms on metabolism of structurally different aldehydes in human liver. Drug Metab. Dispos. 30, 69-73 (2002).

17. Brewer, T. F. \& Chang, C. J. An Aza-cope reactivity-based fluorescent probe for imaging formaldehyde in living cells. J. Am. Chem. Soc. 137, 10886-10889 (2015).

18. Roth, A., Li, H., Anorma, C. \& Chan, J. A reaction-based fluorescent probe for imaging of formaldehyde in living cells. J. Am. Chem. Soc. 137, 10890-10893 (2015).

19. He, L. et al. An ultra-fast illuminating fluorescent probe for monitoring formaldehyde in living cells, shiitake mushrooms, and indoors. Chem. Commun. 52, 9582-9585 (2016).

20. Tang, Y., Kong, X., Xu, A., Dong, B. \& Lin, W. Development of a two-photon fluorescent probe for imaging of endogenous formaldehyde in living tissues. Angew. Chem. Int. Ed. 55, 3356-3359 (2016).

21. Bruemmer, K. J., Brewer, T. F. \& Chang, C. J. Fluorescent probes for imaging formaldehyde in biological systems. Curr. Opin. Chem. Biol. 39, 17-23 (2017).

22. Tang, Y., Ma, Y., Yin, J. \& Lin, W. Strategies for designing organic fluorescent probes for biological imaging of reactive carbonyl species. Chem. Soc. Rev. $\mathbf{4 8}$, 4036-4048 (2019).

23. Fan, Y. C., Makar, M., Wang, M. X. \& Ai, H. W. Monitoring thioredoxin redox with a genetically encoded red fluorescent biosensor. Nat. Chem. Biol. 13, 1045-1052 (2017).

24. Sanford, L. \& Palmer, A. Recent advances in development of genetically encoded fluorescent sensors. Method. Enzymol. 589, 1-49 (2017).

25. Sun, F. M. et al. A genetically encoded fluorescent sensor enables rapid and specific detection of dopamine in flies, fish, and mice. Cell 174, 481-496 (2018).

26. Chen, N. H., Djoko, K. Y., Veyrier, F. J. \& McEwan, A. G. Formaldehyde stress responses in bacterial pathogens. Front. Microbiol. 7, 257 (2016).

27. Yurimoto, H. et al. HxlR, a member of the DUF24 protein family, is a DNAbinding protein that acts as a positive regulator of the formaldehyde-inducible hxlAB operon in Bacillus subtilis. Mol. Microbiol. 57, 511-519 (2005).

28. Galperin, M. Y. Structural classification of bacterial response regulators: diversity of output domains and domain combinations. J. Bacteriol. 188, 4169-4182 (2006).

29. Osman, D. et al. The effectors and sensory sites of formaldehyde-responsive regulator FrmR and metal-sensing variant. J. Biol. Chem. 291, 19502-19516 (2016).

30. Denby, K. J. et al. The mechanism of a formaldehyde-sensing transcriptional regulator. Sci. Rep. 6, 38879 (2016).

31. Busenlehner, L. S., Pennella, M. A. \& Giedroc, D. P. The SmtB/ArsR family of metalloregulatory transcriptional repressors: structural insights into prokaryotic metal resistance. FEMS Microbiol. Rev. 27, 131-143 (2003).

32. Palm, G. J. et al. Structural insights into the redox-switch mechanism of the MarR/DUF24-type regulator HypR. Nucleic Acids Res. 40, 4178-4192 (2012). 
33. Ji, Q. J. et al. Molecular mechanism of quinone signaling mediated through Squinonization of a YodB family repressor QsrR. Proc. Natl Acad. Sci. USA 110, 5010-5015 (2013).

34. Leelakriangsak, M. et al. Regulation of quinone detoxification by the thiol stress sensing DUF24/MarR-like repressor, YodB in Bacillus subtilis. Mol. Microbiol. 67, 1108-1124 (2008).

35. Nagai, T., Sawano, A., Park, E. S. \& Miyawaki, A. Circularly permuted green fluorescent proteins engineered to sense Ca2+. Proc. Natl Acad. Sci. USA 98, 3197-3202 (2001).

36. Ji, Q. J., Zhao, B. S. \& He, C. A highly sensitive and genetically encoded fluorescent reporter for ratiometric monitoring of quinones in living cells. Chem. Commun. 49, 8027-8029 (2013).

37. Tao, R. K. et al. Genetically encoded fluorescent sensors reveal dynamic regulation of NADPH metabolism. Nat. Methods 14, 720-728 (2017).

38. Nguyen, T. T. et al. Genome-wide responses to carbonyl electrophiles in Bacillus subtilis: control of the thiol-dependent formaldehyde dehydrogenase AdhA and cysteine proteinase YraA by the MerR-family regulator YraB (AdhR). Mol. Microbiol. 71, 876-894 (2009).

39. Siegel, D., Meinema, A. C., Permentier, H., Hopfgartner, G. \& Bischoff, R. Integrated quantification and identification of aldehydes and ketones in biological samples. Anal. Chem. 86, 5089-5100 (2014).

40. Keung, W. M. \& Vallee, B. L. Daidzin-a potent, selective inhibitor of human mitochondrial aldehyde dehydrogenase. Proc. Natl Acad. Sci. USA 90 1247-1251 (1993).

41. Barnett, S. D., Smith, C. R., Ulrich, C. C., Baker, J. E. \& Buxton, I. L. O. Snitrosoglutathione reductase underlies the dysfunctional relaxation to nitric oxide in preterm labor. Sci. Rep. 8, 5614 (2018).

42. Umansky, C. et al. Endogenous formaldehyde scavenges cellular glutathione resulting in cytotoxic redox disruption. https://www.biorxiv.org/content/ 10.1101/2020.05.14.090738v1 bioRxiv (2020).

43. Aluise, C. D. et al. Site-specific, intramolecular cross-linking of Pin1 active site residues by the lipid electrophile 4-Oxo-2-nonenal. Chem. Res. Toxicol. 28, 817-827 (2015).

44. Long, M. J. C. et al. Akt3 is a privileged first responder in isozyme-specific electrophile response. Nat. Chem. Biol. 13, 333-338 (2017).

45. Bollong, M. J. et al. A metabolite-derived protein modification integrates glycolysis with KEAP1-NRF2 signalling. Nature 562, 600-604 (2018).

46. Parvez, S., Long, M. J. C., Poganik, J. R. \& Aye, Y. Redox signaling by reactive electrophiles and oxidants. Chem. Rev. 119, 4464-4469 (2019).

47. Pietzke, M. et al. Amino acid dependent formaldehyde metabolism in mammals. Commun. Chem. 3, 78 (2020).

48. Hira, A. et al. Variant ALDH2 is associated with accelerated progression of bone marrow failure in Japanese Fanconi anemia patients. Blood 122, 3206-3209 (2013).

49. Nie, D. et al. Comprehensive analysis on phenotype and genetic basis of Chinese Fanconi anemia patients: dismal outcomes call for nationwide studies. BMC Med. Genet. 21, 118 (2020).

50. Hao, Z. Y. et al. The multiple antibiotic resistance regulator MarR is a copper sensor in Escherichia coli. Nat. Chem. Biol. 10, 21-28 (2014).

51. Gibson, D. G. et al. Enzymatic assembly of DNA molecules up to several hundred kilobases. Nat. Methods 6, 343-345 (2009).

52. Zhang, M. et al. A genetically incorporated crosslinker reveals chaperone cooperation in acid resistance. Nat. Chem. Biol. 7, 671-677 (2011).

53. Wang, Q. S. et al. Upgrade of macromolecular crystallography beamline BL17U1 at SSRF. Nucl. Sci. Tech. 29, 68 https://doi.org/10.1007/s41365-0180398-9 (2018).

54. Otwinowski, Z. \& Minor, W. Processing of X-ray diffraction data collected in oscillation mode. Method Enzymol. 276, 307-326 (1997).

55. Minor, W., Cymborowski, M., Otwinowski, Z. \& Chruszcz, M. HKL-3000: the integration of data reduction and structure solution-from diffraction images to an initial model in minutes. Acta Crystallogr. D. 62, 859-866 (2006).

56. Mccoy, A. J. et al. Phaser crystallographic software. J. Appl. Crystallogr. 40, 658-674 (2007).
57. Winn, M. D. et al. Overview of the CCP4 suite and current developments. Acta Crystallogr. D. 67, 235-242 (2011)

58. Murshudov, G. N., Vagin, A. A. \& Dodson, E. J. Refinement of macromolecular structures by the maximum-likelihood method. Acta Crystallogr. D. 53, 240-255 (1997)

59. Adams, P. D. et al. PHENIX: a comprehensive Python-based system for macromolecular structure solution. Acta Crystallogr. D. 66, 213-221 (2010).

60. Emsley, P., Lohkamp, B., Scott, W. G. \& Cowtan, K. Features and development of Coot. Acta Crystallogr. D. 66, 486-501 (2010).

61. Williams, C. J. et al. MolProbity: more and better reference data for improved all-atom structure validation. Protein Sci. 27, 293-315 (2018).

62. Madeira, F. et al. The EMBL-EBI search and sequence analysis tools APIs in 2019. Nucleic Acids Res. 47, W636-W641 (2019).

\section{Acknowledgements}

We thank the staff members of the Shanghai Synchrotron Radiation Facility (SSRF) and the National Center for Protein Science, Shanghai (NCPSS). This work was supported by research grants from the National Key Research and Development Program of China (2016YFA0501500) and the National Natural Science Foundation of China (21521003, 21740001 and 91753000$)$.

\section{Author contributions}

R.Z. and G.Z. were the key contributor in designing and conducting majority of the experiments. P.R.C. conceived and directed the project. M.J. conducted key experiments. Y.H., J.L., and J.Z. conducted certain experiments. R.Z., G.Z., and P.R.C. wrote the manuscript. Y.L. contributed to experimental design. M.J. and Y.L. edited the manuscript.

\section{Competing interests}

The authors declare no competing interests.

\section{Additional information}

Supplementary information is available for this paper at https://doi.org/10.1038/s41467020-20754-4.

Correspondence and requests for materials should be addressed to P.R.C.

Peer review information Nature Communications thanks Haike Antelmann, Lucas Pontel and the other, anonymous, reviewer(s) for their contribution to the peer review of this work.

Reprints and permission information is available at http://www.nature.com/reprints

Publisher's note Springer Nature remains neutral with regard to jurisdictional claims in published maps and institutional affiliations.

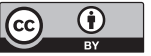

Open Access This article is licensed under a Creative Commons Attribution 4.0 International License, which permits use, sharing, adaptation, distribution and reproduction in any medium or format, as long as you give appropriate credit to the original author(s) and the source, provide a link to the Creative Commons license, and indicate if changes were made. The images or other third party material in this article are included in the article's Creative Commons license, unless indicated otherwise in a credit line to the material. If material is not included in the article's Creative Commons license and your intended use is not permitted by statutory regulation or exceeds the permitted use, you will need to obtain permission directly from the copyright holder. To view a copy of this license, visit http://creativecommons.org/ licenses/by/4.0/.

(C) The Author(s) 2021 